\title{
CXCL10 stabilizes T cell-brain endothelial cell adhesion leading to the induction of cerebral malaria
}

\author{
Elizabeth W. Sorensen, Jeffrey Lian, Aleksandra J. Ozga, Yoshishige Miyabe, Sophina W. Ji, \\ Shannon K. Bromley, Thorsten R. Mempel, and Andrew D. Luster
}

Center for Immunology and Inflammatory Diseases, Division of Rheumatology, Allergy and Immunology, Massachusetts General Hospital, Harvard Medical School, Boston, Massachusetts, USA.

\begin{abstract}
Malaria remains one of the world's most significant human infectious diseases and cerebral malaria (CM) is its most deadly complication. CM pathogenesis remains incompletely understood, hindering the development of therapeutics to prevent this lethal complication. Elevated levels of the chemokine CXCL10 are a biomarker for CM, and CXCL10 and its receptor CXCR3 are required for experimental CM (ECM) in mice, but their role has remained unclear. Using multiphoton intravital microscopy, CXCR3 receptor- and ligand-deficient mice and bone marrow chimeric mice, we demonstrate a key role for endothelial cell-produced CXCL10 in inducing the firm adhesion of T cells and preventing their cell detachment from the brain vasculature. Using a CXCL9 and CXCL10 dualCXCR3-ligand reporter mouse, we found that CXCL10 was strongly induced in the brain endothelium as early as 4 days after infection, while CXCL9 and CXCL10 expression was found in inflammatory monocytes and monocyte-derived DCs within the blood vasculature on day 8 . The induction of both CXCL9 and CXCL10 was completely dependent on IFN- $\gamma$ receptor signaling. These data demonstrate that IFN- $\gamma$-induced, endothelium-derived CXCL10 plays a critical role in mediating the T cell-endothelial cell adhesive events that initiate the inflammatory cascade that injures the endothelium and induces the development of ECM.
\end{abstract}

Authorship note: $\mathrm{AJO}$ and YM contributed equally to this work.

Conflict of interest: The authors have declared that no conflict of interest exists.

Submitted: November 29, 2017

Accepted: March 14, 2018

Published: April 19, 2018

Reference information: JCI Insight. 2018;3(8):e98911. https:// doi.org/10.1172/jci.insight.98911.

\section{Introduction}

Malaria remains one of the world's most important human infectious diseases, with over 200 million new cases and approximately half a million deaths per year, the majority in children $(1,2)$. A deadly complication of this infection is cerebral malaria (CM), which primarily afflicts children under 5 years old, and is characterized by parasitemia, fever, and coma. CM has a mortality rate of $15 \%-30 \%$ and $10 \%-24 \%$ of surviving patients have long-lasting sequelae and neurological impairment (3). There is a mouse model of $\mathrm{CM}$ induced by Plasmodium berghei ANKA (PbA) infection of C57BL/6 mice. Ninety percent of infected mice develop mild neurological symptoms between days 7 and 9 and will die within 24 hours of developing more severe neurological symptoms, which mirror human CM. The pathology of experimental CM (ECM) mirrors human CM in many ways, including brain endothelial cell (EC) activation (4, 5), inflammatory cytokine and chemokine production (6-9), parasitized $\mathrm{RBC}(\mathrm{pRBC})$ or leukocyte sequestration in the brain vasculature, and breakdown of the blood-brain barrier $(10,11)$. pRBC sequestration is thought to be a key pathological feature of human CM; however, its role in murine ECM is more variable and is thought to be dependent on the Plasmodium species used to infect mice (12). ECM is dependent on the presence of $\mathrm{CD}^{+}(13-15)$ and $\mathrm{CD}^{+}(15,16) \mathrm{T}$ cells, and their effector functions, specifically perforin and granzyme $(17,18)$ and IFN- $\gamma(13,16,19,20)$. Endothelial cross-presentation of malarial antigens has been shown to play a role in ECM pathogenesis $(21,22)$, and in vivo imaging data recently revealed that parasite-specific $\mathrm{CD}^{+} \mathrm{T}$ cells interact with luminal antigen within the cerebrovasculature to induce fatal vascular breakdown in ECM (13).

Relevant for our study, high levels of the chemokine CXCL10 were found in the blood and brain in humans that died from CM (23-25). Elevated plasma and cerebral spinal fluid (CSF) CXCL10 levels are one of the best predictors of both CM onset and mortality in children $(25,26)$, and humans with a CXCL10 polymorphism associated with increased CXCL10 levels have increased susceptibility to CM (27). CXCL10 
is induced in cells by type 1 and type II interferons (28) and induces chemotaxis in T cells via activation of CXCR3, a G protein-coupled 7-transmembrane-spanning receptor (29). CXCR3 also binds 2 other interferon-inducible chemokines, CXCL9 (30) and CXCL11 (31), although CXCL11 is a null mutant in C57BL/6 mice (32). The induction of CXCR3 on both $\mathrm{CD} 4^{+} \mathrm{Th} 1$ cells and $\mathrm{CD} 8^{+}$cytotoxic $\mathrm{T}$ lymphocytes (CTLs) occurs rapidly after activation $(33,34)$, and is controlled by the master transcription factor T-bet $(35,36)$. In the murine model, CXCL9 and CXCL10 are highly induced in the spleen and brain of mice before and during the development of ECM. $\mathrm{CxCr}^{-/-}$mice are highly protected from $\mathrm{CM}$, and $\mathrm{CXCR} 3^{+} \mathrm{CD} 8^{+} \mathrm{T}$ effector cells have been shown to mediate CM pathogenesis $(6,7,37)$.

However, the role of CXCR3 in $\mathrm{CD}^{+} \mathrm{T}$ cell function during $\mathrm{CM}$ pathogenesis is not understood. CXCR3 mediates $\mathrm{T}$ cell chemotaxis in vitro (29) and T cell migration in vivo $(38,39)$, and contributes to the accumulation of $\mathrm{T}$ cells in tissue in murine models of type 1 -mediated human diseases $(40,41)$. There is also evidence that CXCR3 contributes to T cell accumulation in the brain in $\operatorname{ECM}(6,37,42)$. Several processes influence $\mathrm{T}$ cell accumulation within tissues, including their entry, retention, and exit from the tissue, as well as their proliferation and contraction within tissue. Some data suggest that CXCR3 can influence all of these processes, including the entry $(38,43)$, contraction $(44-46)$, and retention $(47,48)$ of effector cells, but how CXCR3 contributes to $\mathrm{T}$ cell accumulation in the brain required for the development of ECM is not understood.

Similar to other leukocytes, T cell entry into tissue is a multistep process that begins with the capture of free-flowing cells on the vessel wall, followed by (a) rolling on the vessel wall in the direction of flow, (b) firm arrest on the endothelium, (c) release from arrest, spreading out and crawling in all directions on the vessel to locate a receptive location for (d) transendothelial migration (TEM) to extravasate into the tissue. In the case of $\mathrm{T}$ cell entry into the CNS, there is little rolling, and in some models, such as experimental autoimmune encephalomyelitis (EAE), rolling is not required for T cell extravasation into the brain (49). $\mathrm{T}$ cell arrest is mediated through activation of integrins, which in turn bind to integrin ligands induced on activated ECs. Chemokines are potent activators of integrins and are thought to be important mediators of $\mathrm{T}$ cell arrest (50). This has been demonstrated for several chemokines in vitro, but has only been demonstrated in vivo for CCL21 with naive T cells on lymph node high endothelial venules at homeostasis in naive mice (51). CXCR3 ligands have been shown to induce the arrest of activated $\mathrm{T}$ cells in vitro on IFN- $\gamma$-stimulated ECs $(52,53)$, but have not been demonstrated to perform this function in an in vivo setting. Crawling, the third major step in the multistep cascade, precedes TEM and can vary in length. In EAE, $\mathrm{T}$ cells crawl for long periods of time on the brain endothelium against blood flow before they undergo TEM and enter the brain parenchyma to induce disease (54). In ECM, however, T cell entry into the brain parenchyma is not frequently observed $(6,7)$, and instead, prolonged $\mathrm{T}$ cell interactions with the brain endothelium are thought to be the pathogenic interaction for disease induction $(13,55)$

Here, using CXCR3 receptor- and ligand-deficient mice, bone marrow chimeric mice, and a CXCR3 ligand reporter mouse, we demonstrate a key role for EC-produced CXCL10 in inducing arrest, firm adhesion, and preventing $\mathrm{T}$ cell detachment from the brain vasculature. These CXCL10/CXCR3-mediated T cell-EC adhesive events lead to an inflammatory cascade that injures the endothelium and induces the development of ECM.

\section{Results}

CXCR3 increases $T$ cell-EC interactions in the brain during CM. To study the role of CXCR3 in mediating $\mathrm{T}$ cell interactions with the brain vasculature during ECM, we used multiphoton intravital microscopy (MP-IVM) to image the cortex of brains in live anesthetized mice infected with PbA pRBC (Figure 1A and Supplemental Video 1; supplemental material available online with this article; https://doi. org/10.1172/jci.insight.98911DS1). To visualize endogenous T cells, DPE-GFP and DPE-GFP C $\mathrm{cr}^{3^{-1}}$ mice were injected i.p. with PbA pRBCs. In these mice, GFP is expressed under the control the of CD4 promoter and its distal and proximal enhancers such that all $\mathrm{T}$ cells as well as plasmacytoid DCs and perivascular macrophages are GFP positive $(56,57)$. Using MP-IVM, we found markedly fewer total T cells per field of view (FOV) interacting with the vasculature of DPE-GFP $C \mathrm{Crr}^{-/-}$mice compared with DPE-GFP mice on day 8 or 9 post-infection (p.i.) (Figure 1B). The number of T cells interacting with the blood vessel in uninfected DPE-GFP mice was similar to infected DPE-GFP $C x \mathrm{Cr}^{-/-}$mice, suggesting that the increase in $\mathrm{T}$ cell-endothelium interactions seen in PbA-infected WT mice was mediated by the CXCR3 chemokine system (Figure 1B). 
A

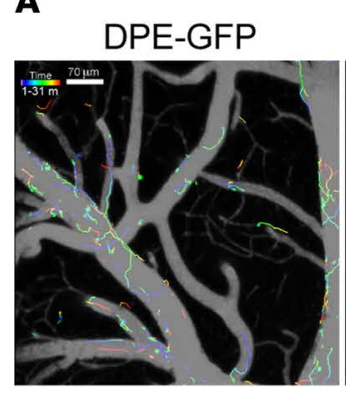

D

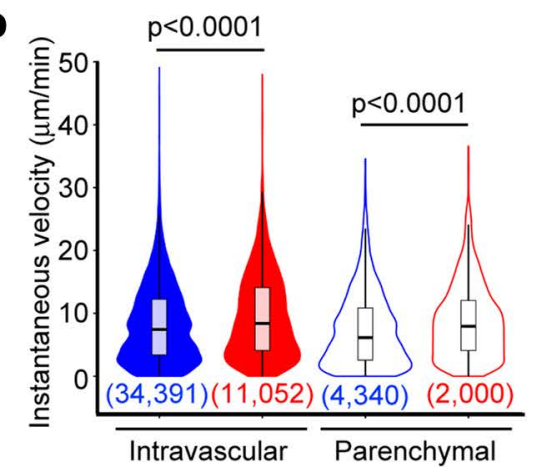

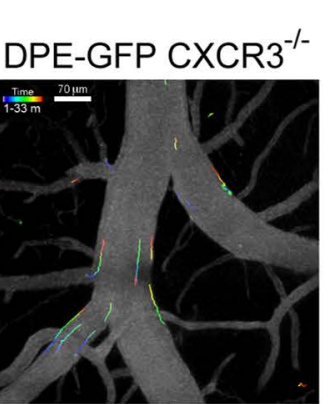

B
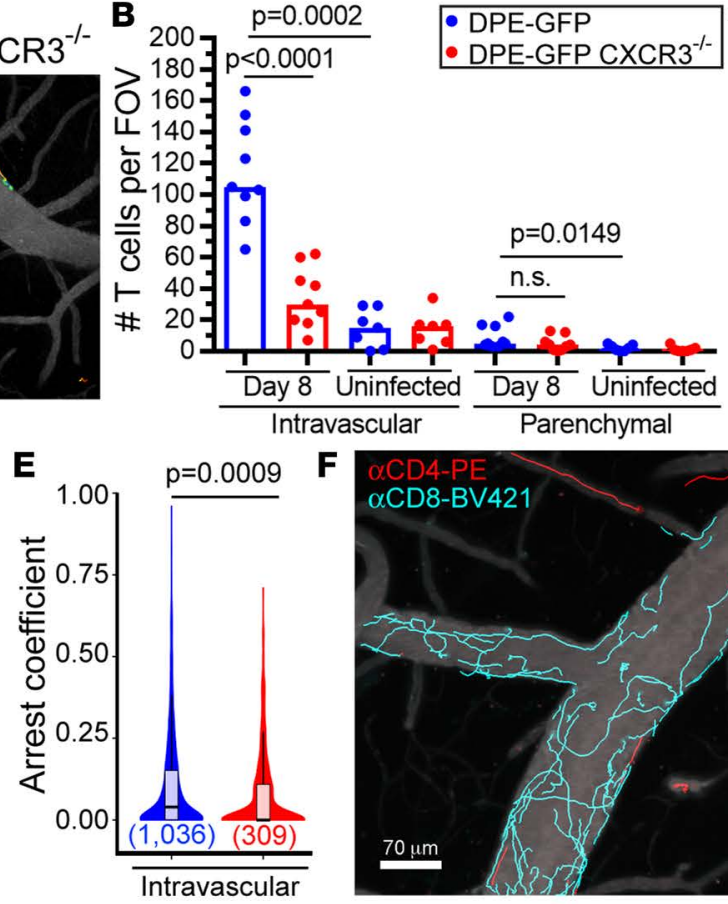

$\mathbf{F}$

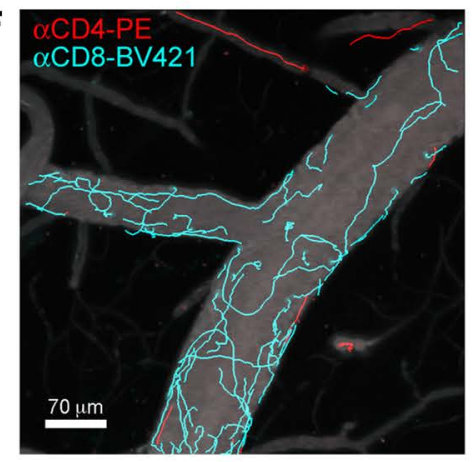

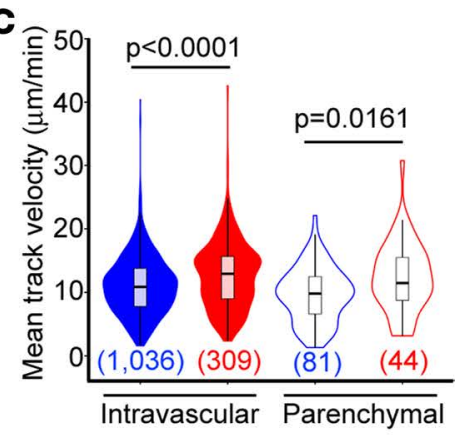

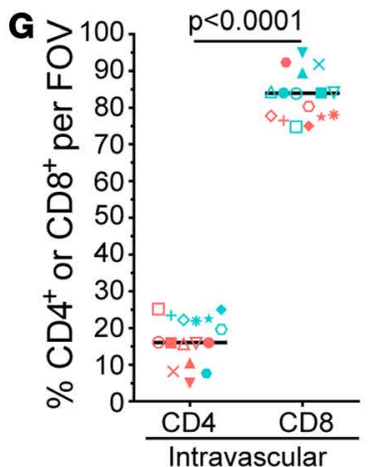

Figure 1. Imaging T cells interacting with the brain vasculature in WT and Cxcr3--- mice. (A) Brains of mice were imaged by multiphoton intravital microscopy with Qtracker 655 (gray) used to reveal patent blood vessels. GFP+ T cells were tracked in the brains of uninfected and day 8 Plasmodium berghei ANKA-infected (PbA-infected) DPE-GFP and DPE-GFP Cxcr3 $3^{-1-}$ mice. The tracks in the example fields of view (FOVs) were color-coded according to the length of time visualized (0 to 30 minutes). GFP+ perivascular macrophages were masked to more clearly visualize the tracked T cells. Scale bars: $70 \mu \mathrm{m}$. See corresponding Supplemental Video 1. (B) Quantification of T cells visible during the entire length of the video, intravascularly and in the brain parenchyma of DPE-GFP (blue symbols) and DPE-GFP $\mathrm{XCr}^{-/-}$(red symbols) uninfected or day 8 infected mice. Each data point is from a different mouse. (C) Mean track velocity, (D) instantaneous 3D velocity, and (E) arrest coefficient of T cells in the brains of DPE-GFP and DPE-GFP $C x C r 3^{-/-}$mice day 8 following $\mathrm{PbA}$ infection. Numbers shown just below the violin plot represent the total number of T cells analyzed in $\mathbf{C}$ and $\mathbf{E}$ and the total number of velocity data points analyzed in $\mathbf{D}$. Velocity measurements were made using Imaris software and arrest coefficient calculated using MATLAB software. The numbers of mice/group total from 3 independent experiments were as follows: infected DPE-GFP $=9$, infected DPE-GFP $C x c r 3^{-/-}=9$, uninfected DPE-GFP $=7$, and uninfected DPE-GFP $\mathrm{CxCr}^{-3^{--}}=7$. (F) Still image displaying the full track length of $\mathrm{CD} 4^{+}$(red) and $\mathrm{CD} 8^{+}$(cyan) T cells interacting with the brain vasculature on day 8 in PbA-infected WT mice during video acquisition. Anti-CD4-PE and anti-CD8-BV421 mAbs were injected retro-orbitally 2 hours prior to imaging. Qtracker 655 (gray) was used to reveal patent blood vessels. Scale bar: $70 \mu \mathrm{m}$. See corresponding Supplemental Video 2. (C) Quantification of the relative numbers of $\mathrm{CD} 4-\mathrm{PE}^{+}$and $\mathrm{CD} 8-\mathrm{BV} 421^{+} \mathrm{T}$ cells as well as $\mathrm{CD} 4-\mathrm{BV} 421^{+}$and $\mathrm{CD} 8-\mathrm{PE}^{+} \mathrm{T}$ cells interacting with the brain vasculature during the entire length of the video. Red symbols = PE-conjugated mAb and cyan = BV421-conjugated mAb. Matching symbols represent data from the same animal. $n=16$ mice total from 6 independent experiments. Groups were compared using either (B-D) Krukal-Wallis with Dunn's multiple comparison tests, (E) Mann-Whitney test, or (G) Wilcoxon's test. Bars or lines represent the median in all plots. Violin plots contain box plots that display the median, 25th and 75th percentiles, and whiskers that represent the $95 \%$ confidence interval.

Tracking the $\mathrm{T}$ cell migratory behavior in the brains of day $8 \mathrm{PbA}$-infected DPE-GFP and DPE-GFP $\mathrm{CxCr}^{-1-}$ mice, we found that intravascular T cells in DPE-GFP $\mathrm{CxCr}^{-/-}$mice interacting with the brain endothelium had increased mean track velocities (Figure 1C) and increased instantaneous velocities (Figure 1D). T cells located in the brain parenchyma of DPE-GFP $\mathrm{CxCr}^{-/-}$mice displayed increased mean track and instantaneous velocity as well. For clarity, we have provided the number of mice and $\mathrm{T}$ cells analyzed per group for all the IVM experiments in Supplemental Table 1. Further analysis revealed that the arrest coefficient (the proportion of time that a cell is arrested while visible) of $\mathrm{T}$ cells attached to the brain vasculature was lower in DPE-GFP $\mathrm{CxCr}^{-/-}$mice (Figure 1E) compared with DPE-GFP mice, further demonstrating the role of CXCR3 in mediating the interaction of T cells with the brain endothelium in ECM. In addition, we observed a marked difference in the crawling behavior of these cells; T cells in DPE-GFP mice were frequently observed meandering, crawling both with and against the direction of blood flow, whereas T cells in DPE-GFP $\mathrm{Cxcr}^{-/-}$mice that interacted with the brain vasculature crawled for shorter amounts of time and, generally, in the direction of flow (Figure 1A and Supplemental Video 1). As both CD4 ${ }^{+}$and $\mathrm{CD}^{+} \mathrm{T}$ cells are $\mathrm{GFP}^{+}$in DPE-GFP mice, we determined the proportion of each that was interacting with the brain vasculature in $\mathrm{PbA}$-infected mice. Fluorescently labeled antibodies against CD4 and CD8 were 
injected retro-orbitally (r.o.) 2 hours prior to imaging WT mice on day 8 after $\mathrm{PbA}$ infection (Figure $1 \mathrm{~F}$ and Supplemental Video 2). We found that approximately $85 \%$ of stained $\mathrm{T}$ cells interacting with the vessels at this time point were $\mathrm{CD}^{+}$, while approximately $15 \%$ were $\mathrm{CD} 4^{+}$, and this was not affected by the fluorochrome-labeled antibodies used to stain $\mathrm{CD}^{+}$and $\mathrm{CD} 8^{+} \mathrm{T}$ cells in vivo (Figure $1 \mathrm{G}$ ).

Since CXCR3 has been demonstrated to play a role in the generation of CD4 ${ }^{+}$Th1-type cells and CD8 ${ }^{+}$ effector/memory cells $(44-46,58)$, we wanted to determine if the generation of effector CD $4^{+}$and CD $8^{+}$ $\mathrm{T}$ cells was influenced by the absence of CXCR3. We therefore analyzed the numbers and phenotype of $\mathrm{CD}^{+}$and $\mathrm{CD}^{+} \mathrm{T}$ cells in the spleen and blood of $\mathrm{WT}$ and $\mathrm{CxCr}^{-1-}$ mice during multiple time points after $\mathrm{PbA}$ infection (Supplemental Figure 1). We found no major differences in either the percentage or number of these populations between WT and $\mathrm{Cxcr}^{-1-}$ mice in the blood (Supplemental Figure 1A) or spleen (Supplemental Figure 1B) during the course of $\mathrm{PbA}$ infection. In addition, we did not find differences in the percentage or number of activated $\left(\mathrm{CD} 44^{+} \mathrm{CD} 69^{+}\right) \mathrm{CD} 4^{+}$and $\mathrm{CD} 8^{+} \mathrm{T}$ cells in blood or spleen during the course of disease between WT and $\mathrm{CxCr}^{-/-}$mice (Supplemental Figure 1, C and D). Further, using intracellular cytokine staining we did not find a difference in the percentage or number of IFN- $\gamma^{+} \mathrm{CD} 4^{+}$and CD $8^{+}$ $\mathrm{T}$ cells in the blood or spleen of WT and $\mathrm{CxCr}^{-1-}$ infected mice on day 0 and on day 8 or 9 p.i. (Supplemental Figure 2, A and B). We also measured the levels of perforin and granzyme mRNA in WT and $C x \mathrm{Cr}^{-1-}$ spleens on days 0,6 , and 8 or 9 p.i. (Supplemental Figure 2, C and D), as these CD ${ }^{+} \mathrm{T}$ cell effector proteins have been implicated in $\operatorname{ECM}(17,18)$. There was no difference between the levels of perforin or granzyme B mRNA in $\mathrm{CxCr}^{-1-}$ and WT mice over the course of infection. However, there were slightly higher levels of perforin in the spleens of $\mathrm{Cxcr}^{-/-}$mice compared with WT mice prior to infection (Supplemental Figure $2 \mathrm{C}$ ). This is possibly due to the increased population of retained central memory $\mathrm{CD} 8^{+} \mathrm{T}$ cells generally found in mice deficient in CXCR3 $(45,46)$.

These data indicated that despite similar numbers and activation levels of circulating T cells in WT and $\mathrm{Cxcr}^{-1-}$ mice, the numbers of $\mathrm{T}$ cells that were arrested and crawling on the brain endothelium and the total length of time that the T cells firmly attached were lower in DPE-GFP $C x \mathrm{Cr}^{-1-}$ mice. This could indicate that $\mathrm{Cxcr}^{-1-} \mathrm{T}$ cells were not able to initiate arrest or were detaching after a short arrest. To distinguish between these possibilities, we observed the frequency of new $\mathrm{T}$ cell attachment and detachment events on the brain endothelium of DPE-GFP and DPE-GFP $\mathrm{CxCr}^{---}$mice (Supplemental Video 3). The images in Figure 2A show a frame before a $\mathrm{T}$ cell of interest becomes visible, followed by the first frame of $\mathrm{T}$ cell attachment and arrest. The subsequent frames then show the fate of these T cells: either firm, long-lasting arrest followed in some cases by crawling, or a short arrest, followed by detachment. T cells in infected DPE-GFP mice had a number of newly attached $\mathrm{T}$ cells that remained attached to the endothelium (Figure 2A, top row, blue arrows). In contrast, attached T cells in infected DPE-GFP $\mathrm{CxCr}^{-1-}$ mice had a tendency to detach from the endothelium after attachment (Figure 2A, bottom row, red and white arrows, respectively), which was not observed in DPE-GFP mice. We enumerated the number of new T cell attachment events as well as the number of newly attached cells that subsequently detached during 121 cycles of acquisition ( 30 -minute observation). We found that there were fewer new $\mathrm{T}$ cell attachment events and more $\mathrm{T}$ cell detachment events in DPE-GFP $C x \mathrm{Cr}^{-/-}$mice compared with DPE-GFP mice on day 8 or 9 p.i. (Figure 2, B and C, closed red vs. blue). It should be stated that although the number of attachment events seen in infected $\mathrm{Cxcr}^{-1-}$ mice was lower than in infected WT mice, CXCR3 does not explain all T cell attachment events during ECM, as we observed more attachment events in infected $\mathrm{CxCr}^{-/-}$mice than in uninfected WT mice (Figure 2B, closed red vs. open blue). Finally, we observed that approximately $15 \%$ of adherent $\mathrm{T}$ cells detached in infected DPE-GFP $\mathrm{Cxcr}^{-/-}$mice, while no adherent T cells detached in infected DPE-GFP mice over approximately 30 minutes of observation (Figure 2D). These detachment events occurred for both arrested and crawling cells. This quantification also revealed that there were more new $\mathrm{T}$ cell attachment events and fewer new $\mathrm{T}$ cell detachment events in infected compared with uninfected mice, yet no differences between uninfected WT and $C x c r 3^{-1-}$ DPE-GFP mice (Figure 2, B and C). Among newly attached T cells, $78 \%$ and $89 \%$ subsequently detached in uninfected DPE-GFP and DPE-GFP $C \mathrm{Crr}^{-1-}$ mice, respectively, compared with $16 \%$ in infected DPE-GFP Cxcr3 ${ }^{-1-}$ mice and $0 \%$ in infected DPE-GFP mice (Figure 2D). These data indicate that CXCR3 has an impact not only on the ability of effector $\mathrm{T}$ cells to be captured by activated endothelium but also has an impact on the strength of adhesion and the conversion to crawling.

Cell-intrinsic defects in adoptively transferred CXCR3-deficient antigen-specific CD8 $8^{+} T$ cells. To elucidate the role(s) for CXCR3 on antigen-specific (Ag-specific) $\mathrm{CD}^{+} \mathrm{T}$ cells, we isolated $\mathrm{CD} 8^{+} \mathrm{T}$ cells from naive Actin-TdTomato $\mathrm{Cxcr}^{+/+}$OT-I and Actin-CFP $\mathrm{Cxcr}^{-/-}$OT-I mice and cotransferred equal numbers into 


\section{A DPE-GFP}
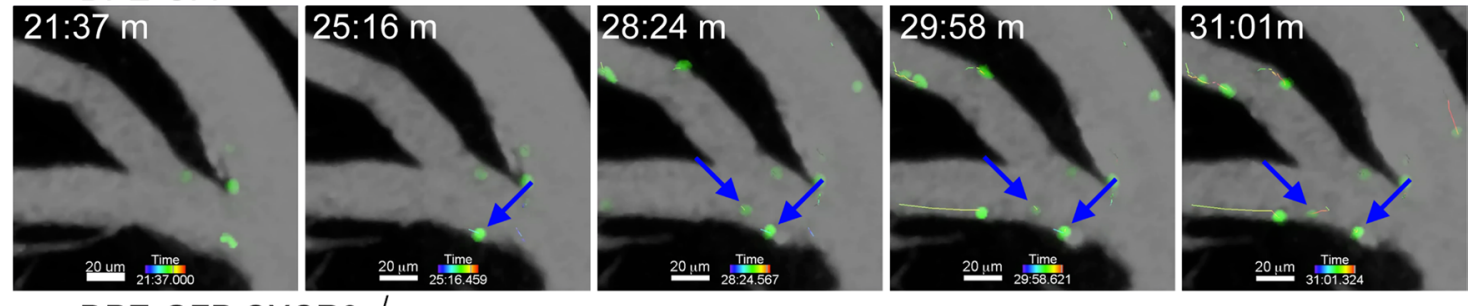

DPE-GFP CXCR3 ${ }^{-1-}$
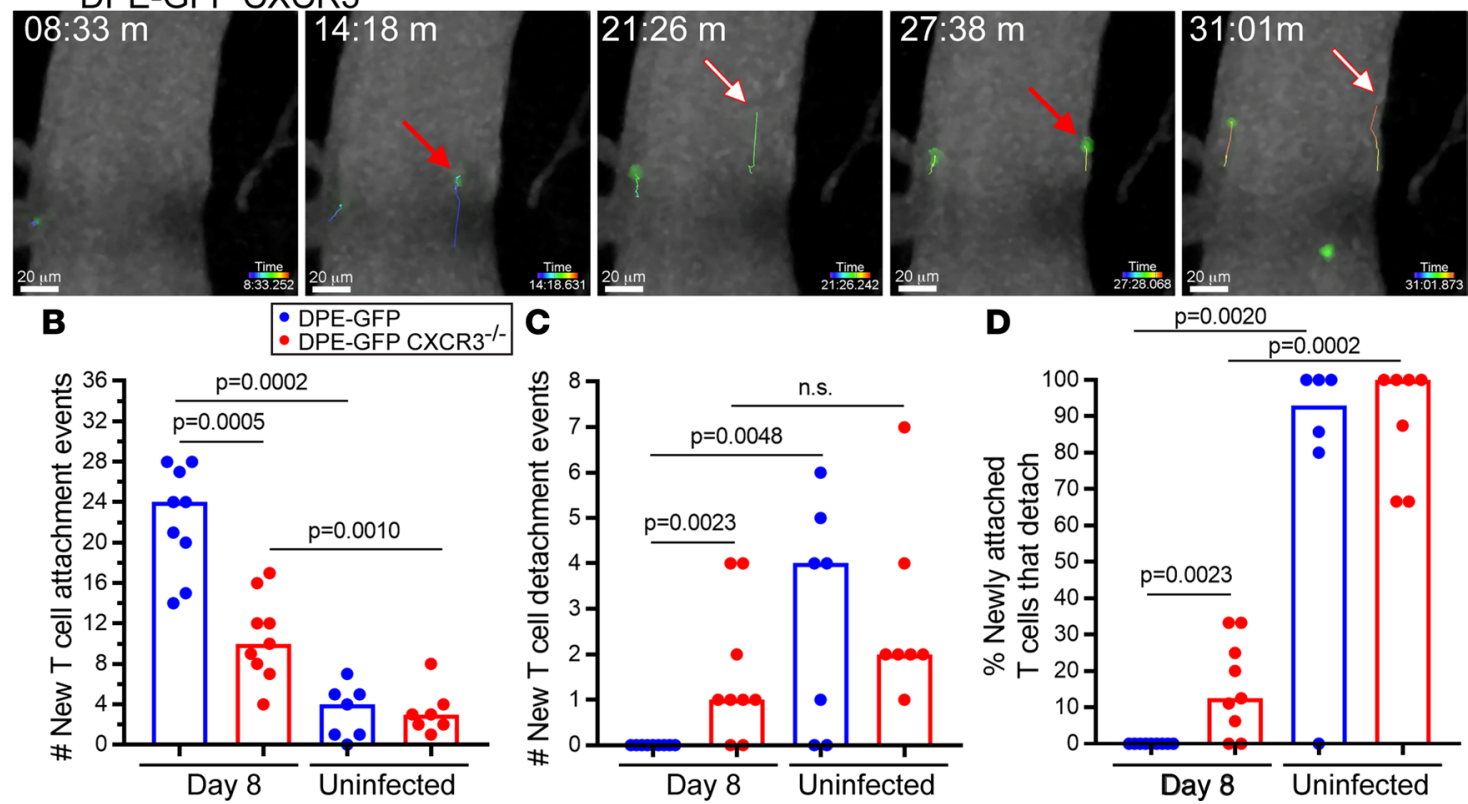

Figure 2. Quantification of T cell attachment and detachment on brain vasculature in WT and $\mathbf{C x c r 3}^{-/-}$mice. Brains of uninfected and day 8 or 9 Plasmodium berghei ANKA-infected (PbA-infected) DPE-GFP or DPE-GFP $\left[\mathrm{XCr}^{-1-}\right.$ mice were imaged using multiphoton intravital microscopy as in Figure 1. (A) Top row shows 2 representative T cells in a PbA-infected DPE-GFP mouse attaching to the brain vasculature for long periods of time and not detaching (blue arrows). Bottom row shows $2 \mathrm{~T}$ cells from the DPE-GFP $C \mathrm{XCr}^{-1-}$ mouse, which attach (red arrows) for a short period and then detach from the endothelium (white arrows). Scale bars: $20 \mu \mathrm{m}$. See corresponding Supplemental Video 3. Number of new T cell (B) attachment and (C) detachment events and (D) percentage of newly attached T cells that subsequently detached during the approximately 30 minutes of data acquisition. The numbers of mice/ group total from 3 independent experiments were as follows: infected DPE-GFP $=9$, infected DPE-GFP $C \times C r 3^{-/-}=9$, uninfected DPE-GFP $=7$, and uninfected DPE-GFP $C \mathrm{xCr}^{-1-}=7$. Groups were compared using Kruskal-Wallis test with Dunn's multiple comparison test. Bars represent the median in all plots.

WT mice. These mice were then infected with ovalbumin-expressing (OVA-expressing) PbA (PbA-OVA) and analyzed on day 8 or 9 (Figure 3A). We found that while an equal number were cotransferred into WT mice before infection, there were only about half as many $\mathrm{Cxcr}^{-1-}$ OT-I cells in the spleen compared with WT OT-I cells (Figure 3B). However, we found that the activation phenotypes of these 2 populations of OT-I cells were similar. The percentage of cells expressing CD25 and CD62L (Figure 3, C and E) was not different between $\mathrm{Cxcr3}^{+/+}$and $\mathrm{Cxcr}^{-/-}$OT-I T cells, and there was only a slight decrease in the percentage of $\mathrm{CD} 69^{+} \mathrm{T}$ cells among $\mathrm{Cxcr3^{-- }}$ OT-I T cells (Figure 3E). Compared with the endogenous WT bulk $\mathrm{CD} 8^{+} \mathrm{T}$ cells, both WT and $\mathrm{Cxcr}^{---} \mathrm{Ag}$-specific populations contained a higher percentage of $\mathrm{CD} 25^{+}$cells and a lower percentage of $\mathrm{CD} 62 \mathrm{~L}^{+}$cells (Figure 3, C and E). As we were interested in the trafficking capability of these cells, we examined the expression level of VLA-4 and LFA-1 on T cells in the spleen by flow cytometry. There was no difference in VLA-4 or LFA-1 expression between WT and $\mathrm{Cxcr}^{-1-}$ OT-I T cells (Figure 3, F and G), and both populations had a higher percentage of VLA- $4^{+}$cells, compared with endogenous bulk $\mathrm{CD} 8^{+} \mathrm{T}$ cells (Figure $3 \mathrm{~F}$ ). In contrast, all endogenous and transferred $\mathrm{CD} 8^{+} \mathrm{T}$ cells expressed LFA-1 (Figure 3G). When we compared the numbers of WT and Cxcr3 ${ }^{-1-}$ OT-I cells in the spleen, blood, and brain, we found a higher ratio ( 3:1) of WT to $\mathrm{Cxcr}^{---}$OT-I cells in the spleen and blood and an even higher ratio (6:1) in the brain (Figure 3H). This indicated that while there was likely a decrease in the ability of the $\mathrm{CxCr}^{---}$OT-I cells to expand in the spleen and subsequently a lower number in the blood, there existed an additional decrease in $\mathrm{Cxcr}^{-/-} \mathrm{Ag}$-specific $\mathrm{CD} 8^{+} \mathrm{T}$ cell accumulation in the $\mathrm{PbA}$-infected brain. 


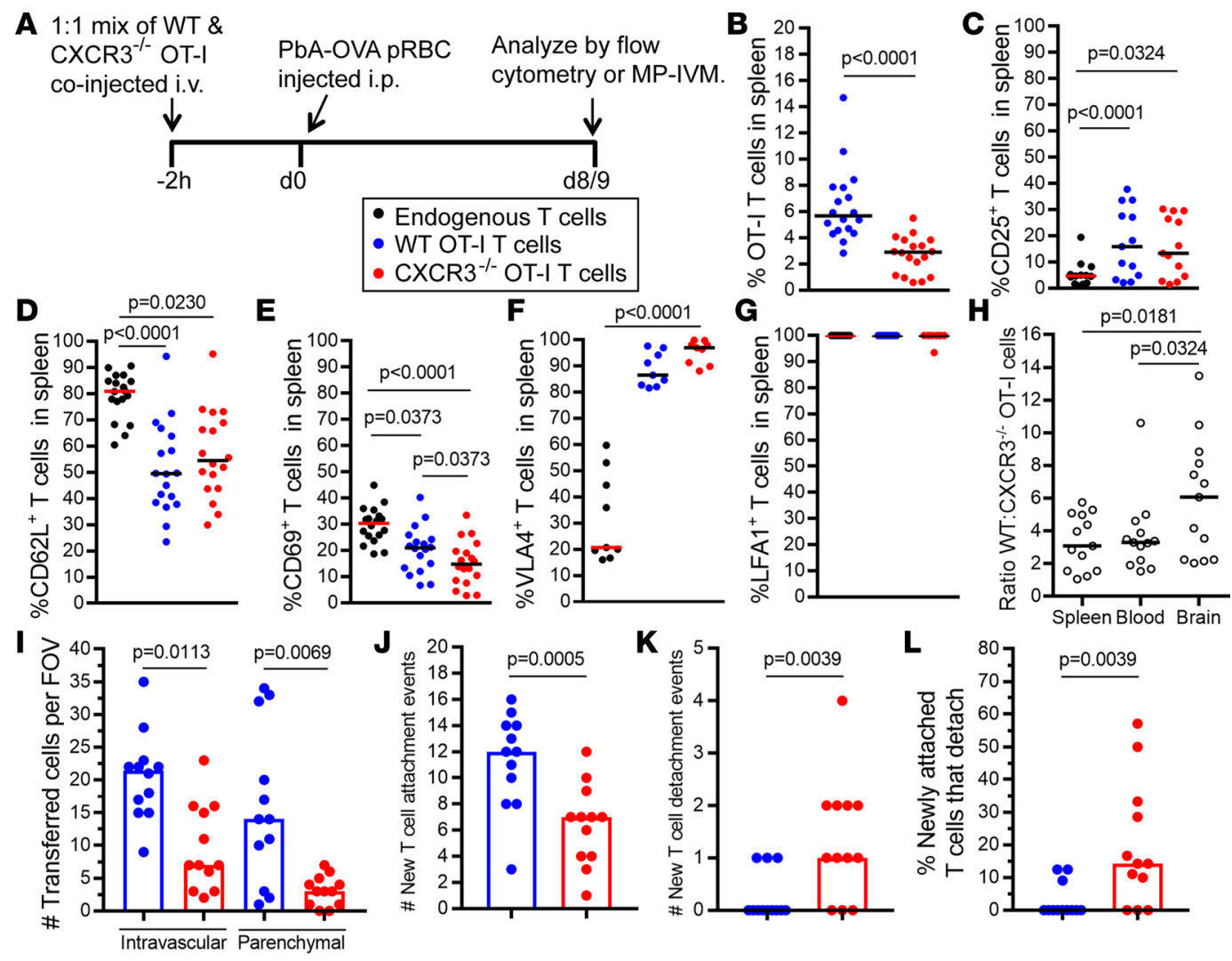

Figure 3. Adoptive transfer of Ag-specific WT and $\mathbf{C x c r 3 ^ { - / - } \text { CD8 }}{ }^{+} \mathbf{T}$ cells. (A) Schematic of experiment. WT mice were coinjected with a 1:1 mix of naive Actin-TdTomato OT-I T cells and Actin-CFP OT-I $\mathrm{Cxcr}^{-/-}$T cells. Mice were infected with ovalbumin-expressing Plasmodium berghei ANKA (PbA-OVA) 2 hours after transfer and analyzed 8 or 9 days later by (B-H) flow cytometry and (I-L) brain multiphoton intravital microscopy (MP-IVM). (B) Percentage of WT OT-I (blue symbols) and CXCr3-/- OT-I cells (red symbols) among total CD8 ${ }^{+}$T cells in the spleen on day 8 or 9 post-infection (p.i.). Percentage of endogenous CD8 ${ }^{+}$T cells (black symbols), WT OT-I, and $\mathrm{CxCr}^{-1-}$ OT-I cells in the spleen stained for (C) CD25, (D) CD62L, (E) CD69, (F) VLA4, or (G) LFA1. (H) Ratio of WT/CXCr3 $3^{-1-}$ OT-I in the spleen, blood, and brain on day 8 or 9 p.i. $n \geq 6$ mice/group from 3 independent experiments. On day 8 or 9 p.i., cotransfer recipients were imaged by MP-IVM. Number of (I) WT OT-I (blue symbols) and Cxcr3-/- OT-I (red symbols) per field of view (FOV) in the brain vasculature and parenchyma and (J) new T cell attachment events, (K) new detachment events, and (L) percentage of newly attached T cells that subsequently detached from the brain vasculature were enumerated using Imaris software. $n=12$ mice total from 4 independent experiments. The groups were compared using (B) Wilcoxon's, (C-I) Friedman's, and (J and K) Krukal-Wallis tests with Dunn's multiple comparison test. Bars and lines represent the median in all plots.

To determine the cell-intrinsic effects of CXCR3 on the migratory behavior of Ag-specific CD8 ${ }^{+} \mathrm{T}$ cells in the setting of full-blown ECM, we again used MP-IVM brain imaging. On day 8 p.i. in PbA-infected WT mice, there were fewer transferred $C x c r 3^{-1-}$ OT-I compared with WT OT-I cells interacting with the brain vasculature or infiltrated into the parenchyma (Figure 3I). As was seen for endogenous T cells in DPE-GFP $\mathrm{CxCr}^{-/-}$mice (Figure 2), Ag-specific Cxcr $3^{-/-} \mathrm{CD}^{+} \mathrm{T}$ cells adoptively transferred into WT mice with ECM displayed decreased attachment (Figure 3J), increased detachment (Figure 3K), and had a higher percentage of newly attached $\mathrm{T}$ cells that detached (Figure $3 \mathrm{~L}$ ) compared with WT Ag-specific CD8 ${ }^{+} \mathrm{T}$ cells cotransferred into the same WT mouse. These data indicate a cell-intrinsic role for CXCR3 in the initial arrest and firm adhesion of $\mathrm{CD} 8^{+} \mathrm{T}$ cells on the brain endothelium in ECM.

Short-term CXCL9 and CXCL10 antibody blockade decreased adhesion and increased T cell detachment from the brain endothelium. To study the effects of acute inhibition of the CXCR3 system in the setting of full-blown ECM, and to begin to elucidate the respective roles of the CXCR3 ligands, we used a short-term antibody blockade experimental protocol. In these experiments, we imaged the brains of DPE-GFP mice (Figure 4, "Pre") and then injected anti-CXCL9 or anti-CXCL10 mAbs r.o., alone or in combination, or an IgG control (Figure 4, "IgG Ctrl"), and then re-imaged the same FOV 4 hours later (Figure 4, "Post"). The strength of T cell-EC adhesion (arrest coefficient) was decreased with CXCL10 mAb treatment (Figure 4A, black violin) and further decreased with a combination of CXCL9 and CXCL10 mAbs (Figure 4A, red violin). However, the treatment with CXCL9 antibody alone had no significant effect on the arrest coefficient (Figure 4A, gray violin). Antibody treatment, either individually or in combination, did not alter the number of new 
A

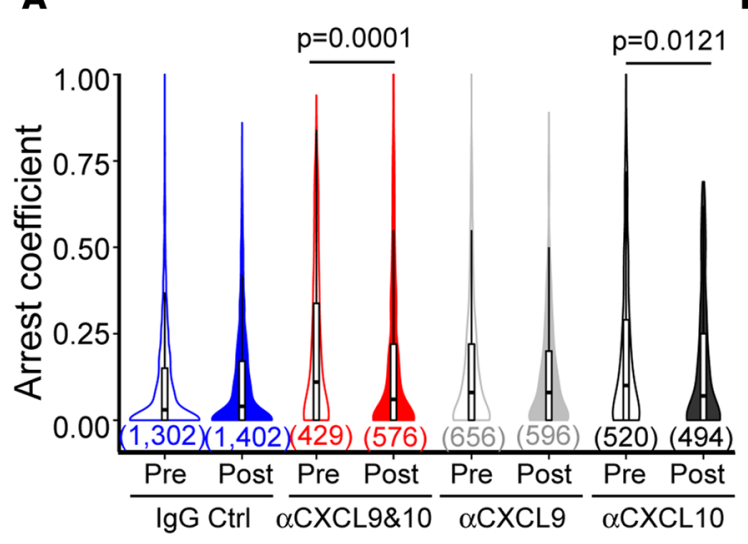

B

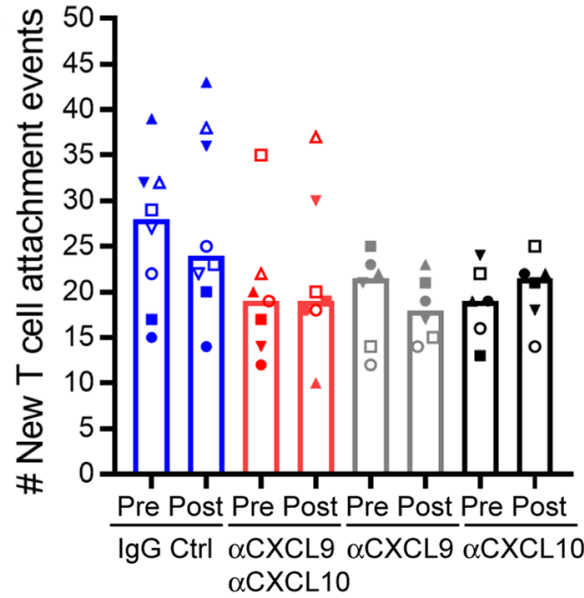

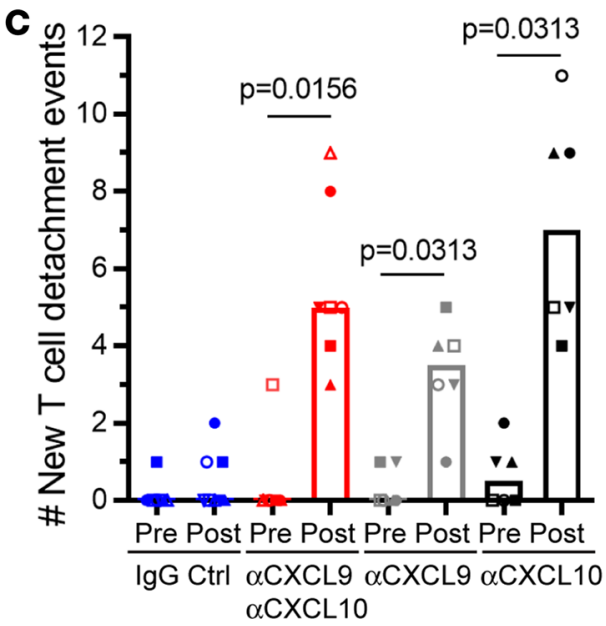

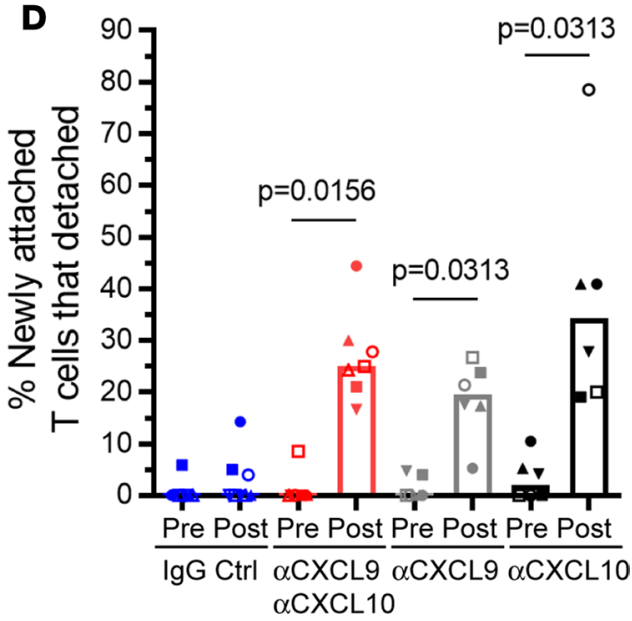

Figure 4. The effects of short-term CXCL9 and CXCL10 blockade on T cell-endothelial cell interactions. After imaging the brains of day 8 Plasmodium berghei ANKA-infected (PbA-infected) DPE-GFP mice (labeled "Pre"), mice were injected with IgC control (blue symbols), both CXCL9 and CXCL10 mAbs (red symbols), CXCL9 mAb alone (gray symbols), or CXCL10 mAb alone (black symbols). Four hours after treatment, the same brain fields of view (FOVs) were re-imaged by multiphoton intravital microscopy (MP-IVM) (labeled "Post"). (A) T cell arrest coefficients pre- and post-treatment were enumerated using Imaris and MATLAB. Violin plots contain box plots that display the median, 25th and 75th percentiles, and whiskers that represent the $95 \%$ confidence interval. Number of new (B) attachment and (C) detachment events was counted manually using Imaris and the (D) percentage of newly attached T cells that subsequently detached was calculated. Matching symbols in scatterplots represent the paired observations from the same mouse and the same field of view pre- and post-treatment. The numbers of mice/group total from 3 independent experiments were as follows: hamster IgG $=8, \alpha C X C L 9 / 10=6, \alpha C X C L 9=6$, and $\alpha C X C L 10=6$. Numbers shown just below the violin plots in $\mathbf{A}$ represent the total number of T cells analyzed. The groups were compared using (A) Mann-Whitney $t$ test and (B-D) Wilcoxon's test. Bars represent the median in all plots.

T cell attachment events (Figure 4B); however, it did result in an increase in the number of T cell detachment events (Figure 4C). In addition, blockade of CXCR3 ligands, alone or in combination, increased the percentage of adherent $\mathrm{T}$ cells that detached in the setting of ECM, although CXCL10 blockade increased the percentage detachment more than CXCL9 blockade: $35 \%$ versus $17 \%$, respectively (Figure 4D). Taken together, these data indicate that CXCL10 plays a role in strengthening T cell-EC adhesion.

Differential expression of CXCL9 and CXCL10 in the brain during ECM. Whole-mount microscopy of CXCR3ligand (REX3) reporter mice (CXCL9 promoter-driven red fluorescent protein [RFP] expression and CXCL10 promoter-driven blue fluorescent protein [BFP] expression) was used to elucidate the cellular sources and pattern of CXCR3 ligand expression in the brain during ECM. Unfixed pieces of brain cortex were stained with directly fluorochrome-conjugated antibodies to visualize EC (anti-CD31) and immune cells (anti-CD45), and imaged using an upright widefield fluorescence microscope. In the uninfected mouse cortex, there was no detectible CXCL9 (RFP) or CXCL10 (BFP) expression (Figure 5, A and B, top rows). However, on day 8 p.i., CXCL10 was highly expressed in CD $31^{+}$blood ECs (Figure 5A, bottom row), and in CD45 $5^{+}$cells (Figure 5B, bottom row, arrow heads). We found that the highest levels of CXC10-BFP were likely in venules, which can be differentiated from arterioles through their larger caliber, rougher luminal endothelial surface and, based 
A
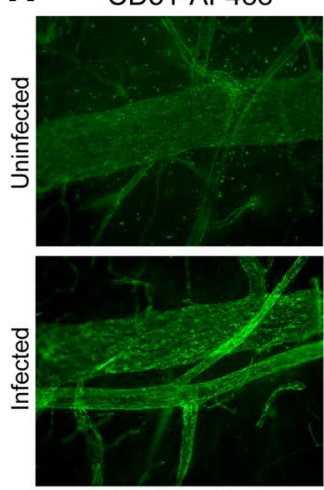

B
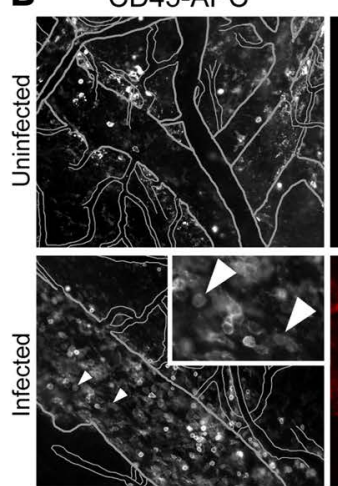

CXCL9-RFP
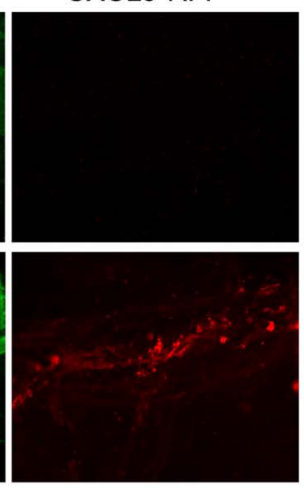

CXCL9-RFP
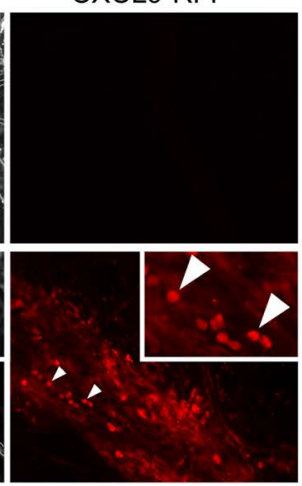

CXCL10-BFP
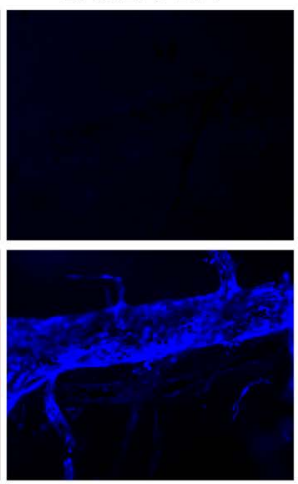

CXCL10-BFP
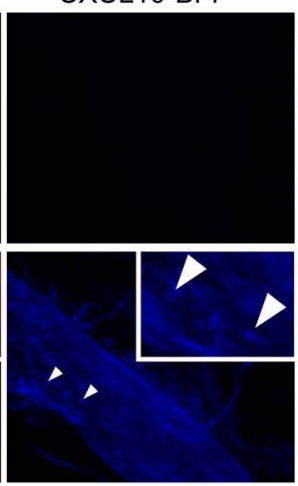

Composite
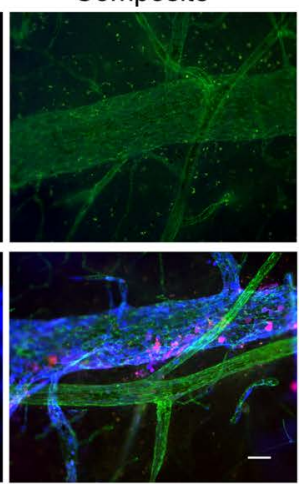

Composite
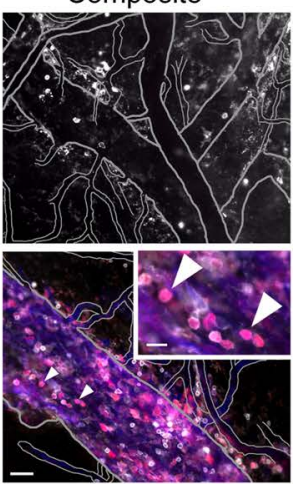

Figure 5. Whole-mount microscopy of brains from CXCL9-RFP and CXCL10-BFP dual reporter (REX3) mice. Whole-mount immunofluorescence was performed on the brain cortices of uninfected or day 8 Plasmodium berghei ANKA-infected (PbA-infected) REX3 mice, where CXCL9-RFP is red and CXCL10-BFP is blue. Representative images are shown stained with (A) anti-CD31-AF488 (green) or (B) anti-CD45-APC (white). In panel B, gray lines represent the outline of blood vessels as determined by CD31 staining and arrowheads in insets indicate CD45, CXCL9, and CXCL10 triple-positive cells. Scale bars: $50 \mu \mathrm{m}$ and $25 \mu \mathrm{m}$ (inset). $n=6$ mice/group total from 3 independent experiments.

on their lower perivascular smooth muscle content, reduced autofluorescence compared with arterioles when viewed under high gain by either whole-mount or 2-photon microscopy $(59,60)$. A CXCL10-BFP-negative arteriole can be seen immediately below the morphologically distinct, large, CXCL10-BFP-bright venule in the bottom row of Figure 5A. CD45 ${ }^{+}$CXCR3 ligand-producing cells were seen in and around the CXCL10$\mathrm{BFP}^{+}$postcapillary venules; no $\mathrm{CD} 45^{+}$cells were detected in CXCL10-BFP-negative venules or arterioles (Figure 5A, bottom row). Interestingly, CXCL10-BFP ${ }^{+}$blood vessels were not detected in other organs during ECM, including in the vessels of the pancreas, liver, and lung (Supplemental Figure 3). CXCL9-RFP was only seen rarely and at low levels in the brain endothelium (Figure 5B, bottom row). However, CXCL9-RFP was seen in a subset of $\mathrm{CD} 45^{+}$cells in and around the vasculature. These $\mathrm{CD} 45^{+}$cells were positive for both CXCL9 and CXCL10 expression (Figure 5B, arrow heads). These data suggest that CXCL10 is the main CXCR3 ligand expressed by the brain endothelium in ECM, while CXCL9 and CXCL10 are produced by $\mathrm{CD} 45^{+}$immune cells in and around the vasculature. This pattern of expression supports a specific and unique role for CXCL10 in T cell capture and adhesion strength along the brain endothelium.

We found that the vast majority of CXCL9-RFP/CXCL10-BFP double-positive CD45 cells were located within the lumen of the vessels. When an anti-CD45 antibody was injected r.o. prior to sacrifice to label only immune cells within the vessel lumen, CXCR3 ligand-producing immune cells were identified within CD31+ vessels (Supplemental Figure 4A). To stain immune cells both in the parenchyma as well as within the vessel lumen, the same tissue was stained ex vivo with an anti-CD45.2 antibody conjugated to a different fluorochrome. Very few immune cells outside of the vessel lumen (unlabeled by the injected CD45 antibody) expressed CXCR3 ligands (Supplemental Figure 4B). These data show that in the infected brain cortex, CXCL9/10 double-positive cells were CD45 ${ }^{+}$and within the vessel lumen (Supplemental Figure $4 \mathrm{~A})$, and the majority of total immune cells in the infected brain cortex were within the vessel lumen or immediately perivascular (Supplemental Figure 4B).

To more clearly follow the induction of EC CXCL10 expression over time, we generated bone marrow chimeric mice in which DPE-GFP bone marrow cells were injected into lethally irradiated REX3 recipients. The presence of GFP on T cells was used to determine the level of reconstitution. Chimeric mice were injected with an intravascular marker (gray) and imaged by MP-IVM on days 4, 6, and 8 after PbA infection (Supplemental Figure 5). Due to the high level of radiation received by these animals, there was 
A

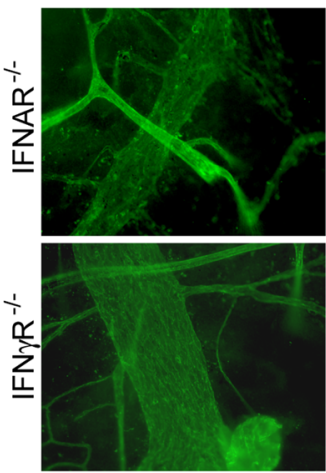

B Brain

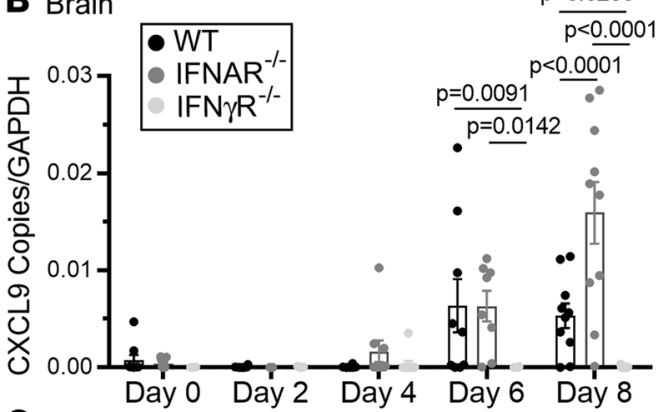

C Spleen

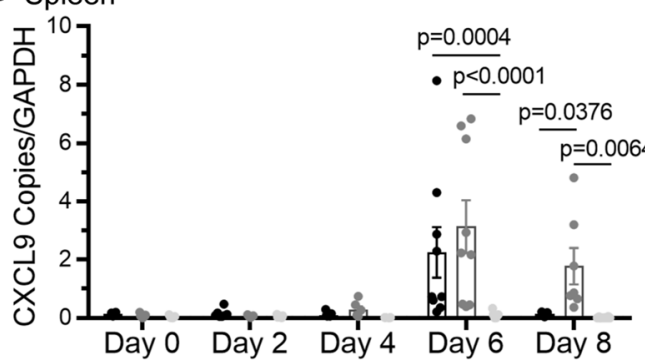

CXCL10-BFP
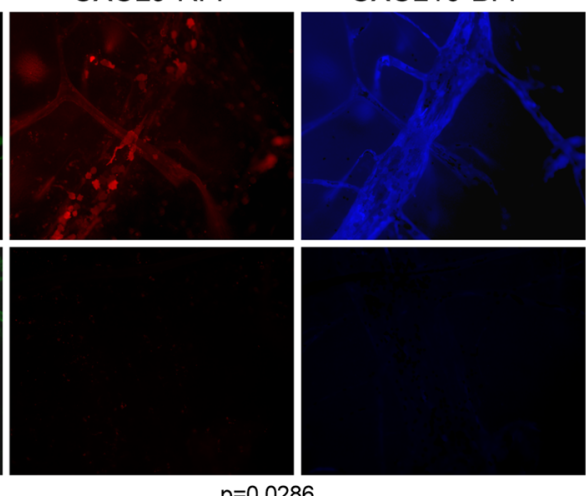

CD45-APC
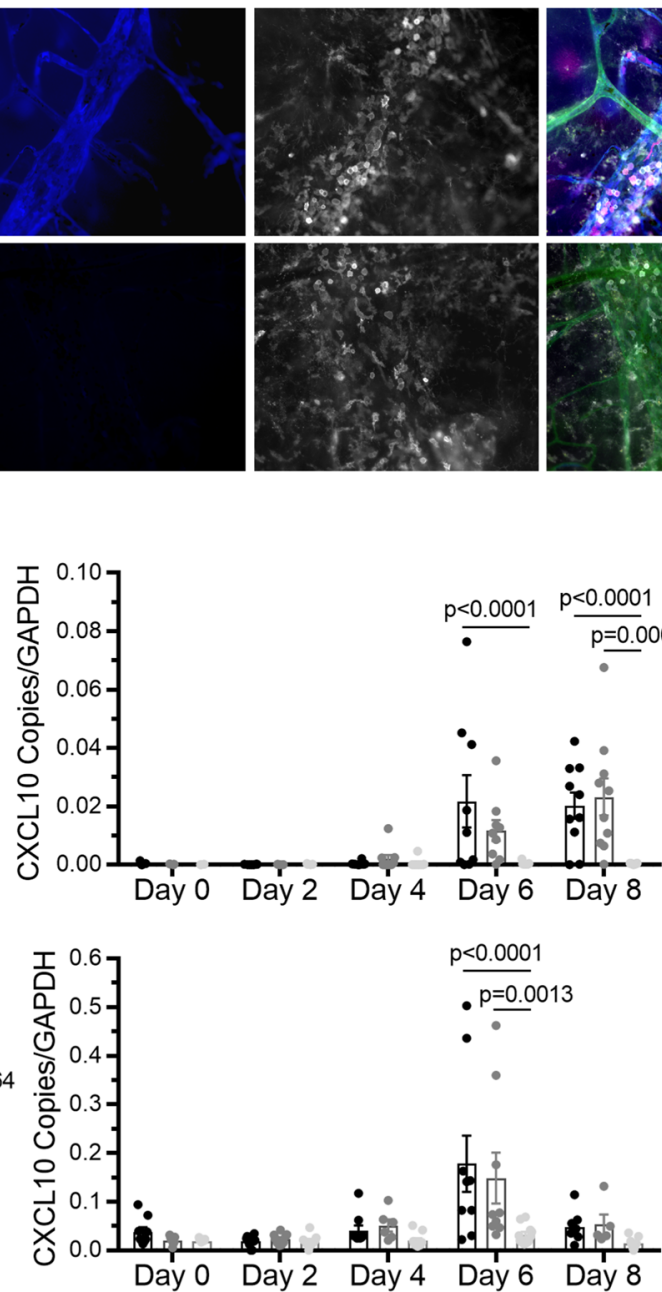

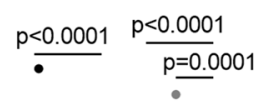

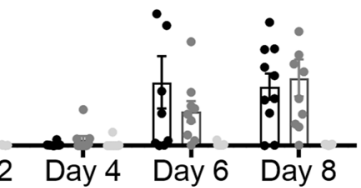

Composite

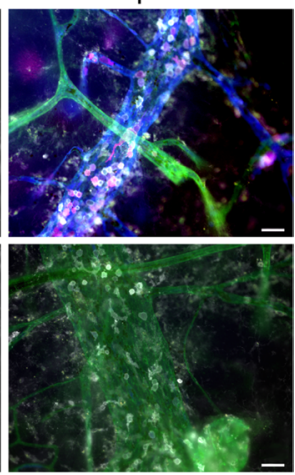
.

Figure 6. CXCL9 and CXCL10 induction is dependent on IFN- $\gamma$ R. (A) REX3-Ifnar $r^{--}$(IFNAR ${ }^{-1-}$, top row) and REX3-Ifngr $r^{-1}$ (IFN $\gamma \mathrm{R}^{-/-}$, bottom row) were infected with Plasmodium berghei ANKA ( $\mathrm{PbA}$ ) and brain cortices were analyzed by whole-mount immunofluorescence on day 8 post-infection (p.i.). Representative images are shown stained with anti-CD31-AF488 (green) and anti-CD45-APC (white). CXCL9-RFP is red and CXCL10-BFP is blue. Scale bars: $50 \mu$ m. $n=6$ mice/ group total from 3 independent experiments. (B and C) qPCR analysis of CXCL9 and CXCL10 RNA levels relative to GAPDH in the (B) brains and (C) spleens of PbA-infected WT (black bars), Ifnar ${ }^{-1}$ (gray bars), and Ifngr ${ }^{-1}$ (black bars) mice on the indicated days p.i. The numbers of mice/group total from 3 independent experiments are listed in day order (i.e., day 0, 2, 4, 6, 8). Brain CXCL9: WT $n=9,8,12,9$, 10; Ifnar ${ }^{-/} n=9,9,9,8,10$; Ifngr ${ }^{-/} n=9,9,11,9,11$. Brain CXCL10:

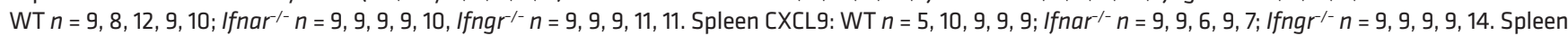
CXCL10: WT $n=9,10,9,9$, 9; Ifnar ${ }^{-1-} n=9,9,6,9,5 ;$ Ifngr $r^{-1-} n=9,9,9,11,11$. The groups in B-D were compared using 2-way ANOVA with Bonferroni's multiple comparison test. Bars represent the mean with SEM in all plots.

a higher level of autofluorescence seen in these images (61), as evidenced by the punctate areas of fluorescence that were present in all 4 fluorescence acquisition channels (GFP is not shown), and appears light pink or white in the composite. This punctate fluorescence is most obvious in the CXCL9-RFP images, since there was very little CXCL9 promoter activity (red) in the stromal compartment in the superficial cortex of these mice. CXCL10-BFP expression (blue) was first detected very faintly or not at all on day 4 p.i. in larger vessels. By day 6, CXCL10-BFP was very bright in large vessels and was also detected in some smaller vessels (Supplemental Figure 5). As these images were taken from MP-IVM videos, we were able to determine the direction of blood flow from the circulating intravascular $\mathrm{GFP}^{+} \mathrm{T}$ cells. Of interest, the image from day 6 (Supplemental Figure 5, middle row) shows a larger CXCL10-BFP-positive venule (blue arrow) crossed horizontally by a CXCL10-BFP-low to -negative arteriole (red arrow). These data corroborate the CXCL10-BFP expression pattern seen in venous blood vessels by whole-mount microscopy (Figure 5A). By day 8, CXCL10-BFP was very bright in most venous blood vessels, including capillaries (vessels with a diameter of $10 \mu \mathrm{m}$ or smaller, white arrow) (Supplemental Figure 5, bottom row). 
A
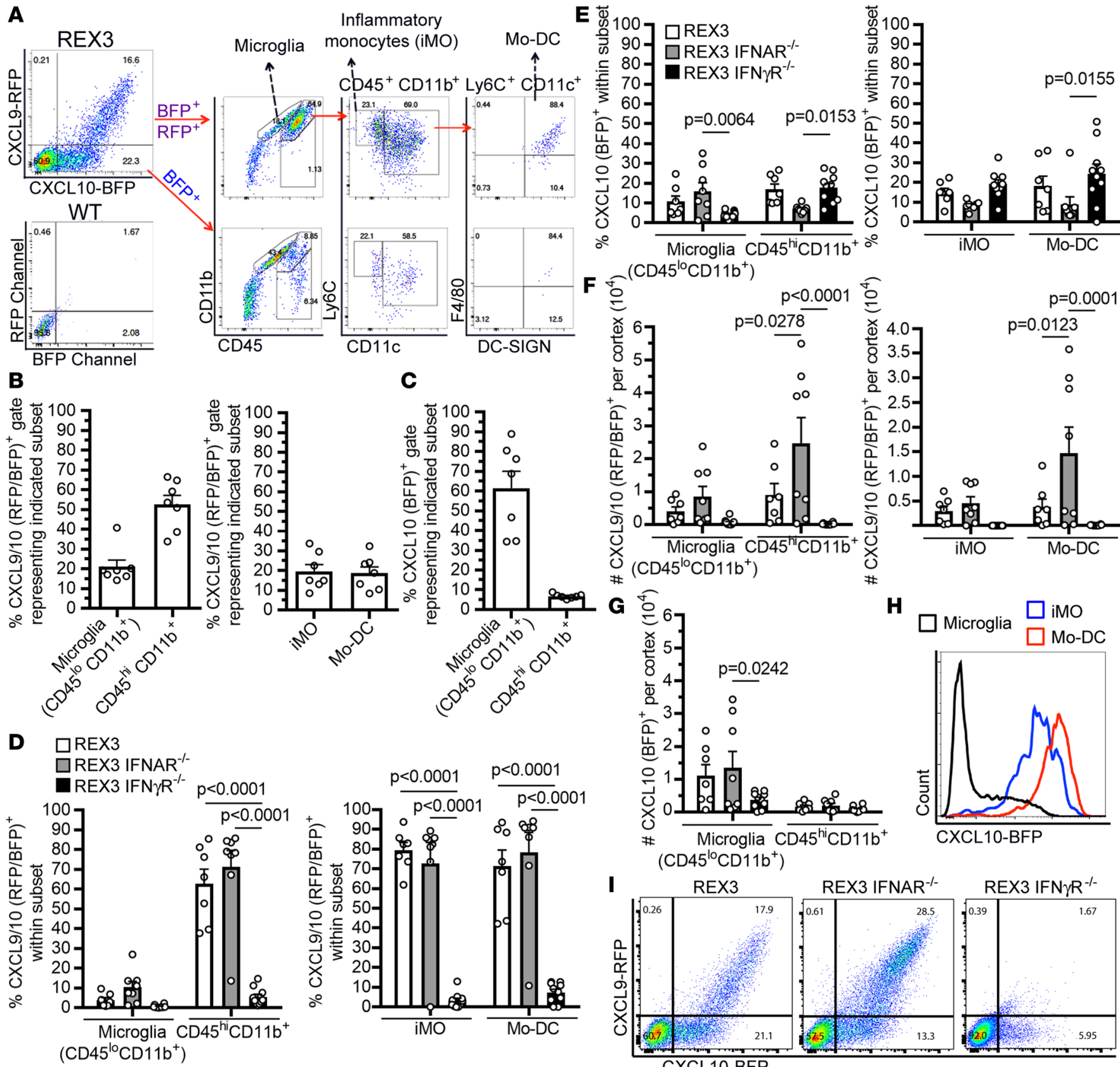

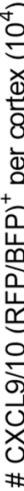
\#
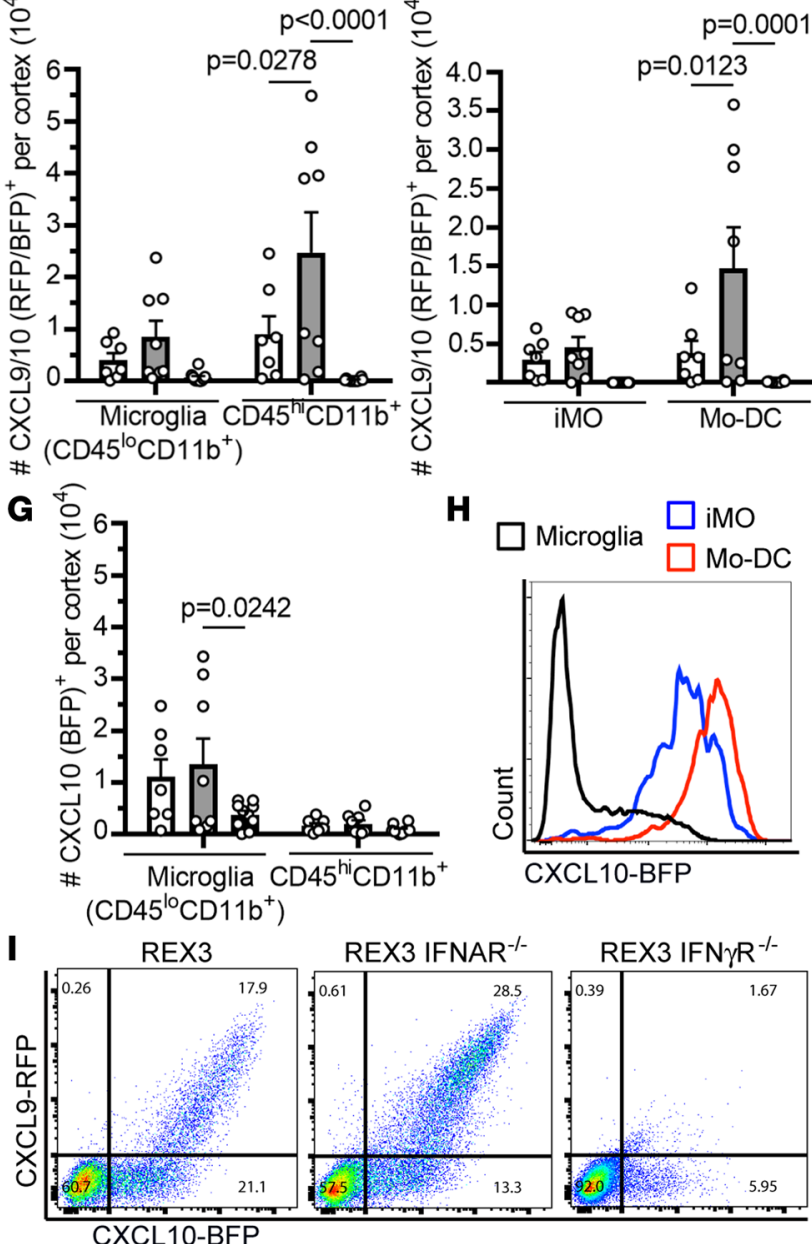

Figure 7. CXCL9 and CXCL10 expression and regulation in immune cells in PbA-infected brains. REX3 (white bars), REX3-Ifnar ${ }^{-1-}$ (IFNAR ${ }^{-1-}$, gray bars), REX3Ifngr $r^{-/-}$(IFN $\gamma \mathrm{R}^{-/-}$, black bars), and WT mice were infected with Plasmodium berghei ANKA (PbA) and on day 8 or 9 single-cell suspensions of brain cortices (Percoll-enriched for leukocytes) were analyzed by flow cytometry. (A) The left most dotplots are from REX 3 and WT cortex single-cell suspension and are stained with viability dye and gated on live single cells only. The gating strategy shown is used in panels $\mathbf{B}$ and $\mathbf{C}$ and also shows the markers used to select the immune subsets shown panels D-G. Percentage of (B) CXCL9 and CXCL10 (RFP+BFP+) double-positive or (C) CXCL10 (BFP+) single-positive cells within each immune cell subset in REX3 mice. Percentage of each indicated subset expressing (D) CXCL9 and CXCL10 (RFP+BFP+) or (E) CXCL10 only (BFP+). Number of cells in each indicated subset expressing (F) CXCL9 and CXCL10 (RFP+BFP+) or (C) CXCL10 only (BFP+) per brain. (H) Histogram depicting the CXCL10-BFP levels in microglia (black line), inflammatory monocytes (iMOs) (blue line), and monocyte-derived DCs (Mo-DCs) (red line). (I) Dot plots showing CXCL9-RFP and CXCL10-BFP expression on Percoll gradient-enriched leukocytes isolated from the brains of REX3, REX3-Ifnar ${ }^{-1 /}$, and REX3-Ifngr $r^{-1-}$ mice. In B-G, all graphs are gated on all live cells. The numbers of mice/group total from 3 independent experiments were as follows: REX3 $=7, \mathrm{REX} 3-\mathrm{Ifnar} \mathrm{r}^{-1-}=8$, and REX3-Ifngr ${ }^{1-}=10$. The groups in D-G were compared using 2-way ANOVA with Bonferroni's multiple comparison test. Bars represent the mean with SEM in all plots.

CXCL9 and CXCL10 production was dependent on IFN- $\gamma$. Type I and II interferon signaling is required for the development of ECM. Deficiency in IFNAR, the receptor for IFN- $\alpha / \beta$, has been reported to provide partial to full protection from the development of ECM (62-64), while deficiency in IFN- $\gamma$ R, the IFN- $\gamma$ receptor, consistently has been reported to provide full protection against the development of ECM (13, 

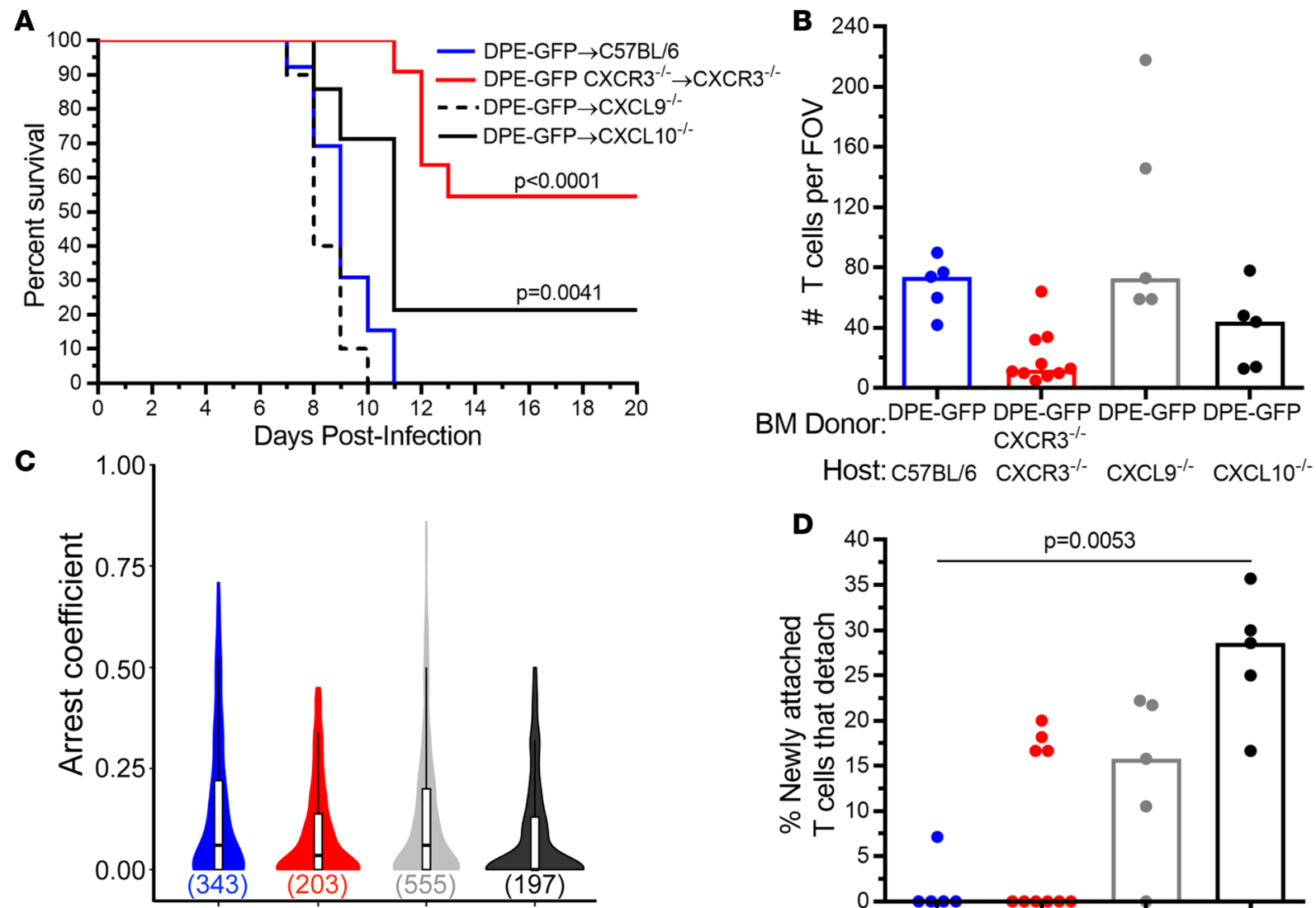

BM Donor: DPE-GFP DPE-GFP DPE-GFP DPE-GFP

Host: C57BL/6 $\mathrm{CXCR}^{-1-}$ CXCL9 ${ }^{-1-} \mathrm{CXCL} 10^{-1-}$

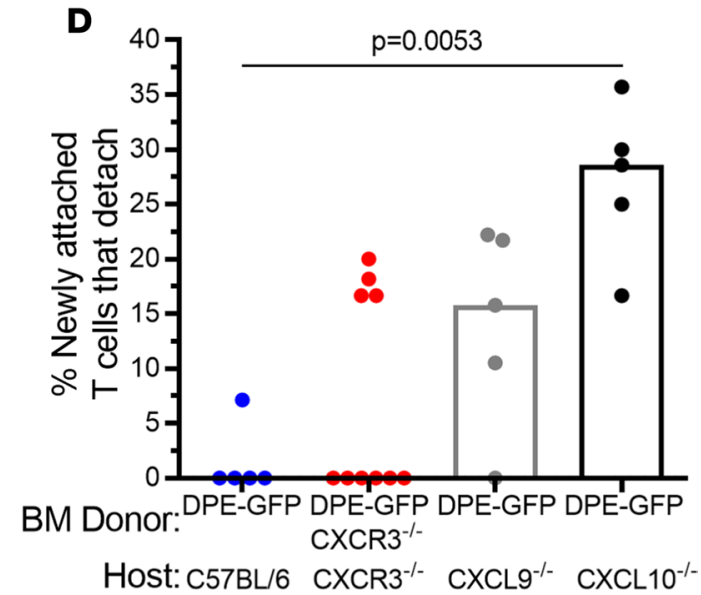

Figure 8. Stromally produced CXCL10 influences survival and T cell interaction with the brain vasculature in PbA-infected mice. Lethally irradiated WT,

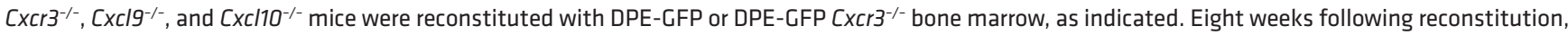
chimeric mice were infected with Plasmodium berghei ANKA (PbA). (A) Kaplan-Meier survival curve. The numbers of mice/group total from 3 independent

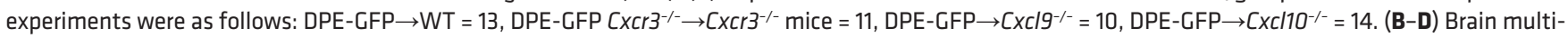
photon intravital microscopy (MP-IVM) of chimeric mice on day 8 or 9 after infection. (B) Number of T cell tracks per field of view (FOV) was analyzed using Imaris software and (C) arrest coefficient was calculated using MATLAB. Violin plots contain box plots that display the median, 25th and 75th percentiles, and whiskers that represent the $95 \%$ confidence interval (note the median of the DPE-GFP $\rightarrow\left[x \mathrm{C} / 10^{-/-}\right.$chimeras is 0 ). Numbers of new T cell attachment and detachment events were manually counted and the (D) percentage of newly attached T cells that subsequently detached were calculated. The numbers of

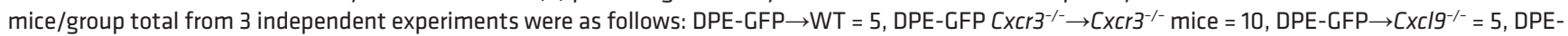
$\mathrm{GFP} \rightarrow\left[\mathrm{Cx} / 10^{-1-}=5\right.$. Numbers shown just below the violin plot in $\mathbf{C}$ represent the total number of T cells analyzed. Groups were compared using either (A) log-rank (Mantel-Cox) test or (B-D) Kruskal-Wallis with Dunn's multiple comparison test. Bars represent the median in all plots. BM, bone marrow.

$16,19,20)$. As IFN- $\alpha / \beta$ and IFN- $\gamma$ can induce the production of CXCL9 and CXCL10 $(28,30)$, we used whole-mount microscopy to examine CXCR3 ligand expression in the brains of REX3-Ifnar ${ }^{\prime-}$ and REX3Ifngr ${ }^{-1}$ mice. We found that there was no difference in the pattern of CXCL9-RFP and CXCL10-BFP expression in infected REX3-Ifnar ${ }^{-1}$ mice compared with infected REX3 mice (Figure 6A, top row vs. Figure 5). In contrast, there was a complete absence of CXCL9-RFP and CXCL10-BFP expression in infected REX3-Ifngr ${ }^{\prime-}$ mice (Figure 6A, bottom row). The dependence of CXCR3 ligand induction on IFN- $\gamma \mathrm{R}$ signaling was confirmed by qPCR of the cortices of WT, Ifnar ${ }^{\prime-}$, and Ifngr ${ }^{\prime-}$ mice (Figure 6B). The induction of CXCL9 and CXCL10 mRNA was increased in the brain by day 6 in WT (white bars) and Ifnar ${ }^{-1}$ (gray bars), but not in Ifngr ${ }^{-1}$ (black bars) mice (Figure 6B). Both CXCL9 and CXCL10 mRNA transcripts were also upregulated in the spleen on day 6 in WT and Ifnar ${ }^{\prime-}$ mice, but not in Ifngr ${ }^{\prime-}$ mice (Figure 6C). Interestingly, CXCL9 and CXCL10 induction was increased to even higher levels in the brains and spleen of Ifnar ${ }^{\prime-}$ mice compared with WT mice. These data demonstrate that the induction of both CXCL9 and CXCL10 in the brains and spleens of PbA-infected mice was completely dependent on IFN- $\gamma$ receptor signaling and not on IFN- $\alpha / \beta$ receptor signaling. 
Inflammatory monocytes and monocyte-derived DCS are the predominant CXCL9- and CXCL10-producing immune cells in the PbA-infected brain. The phenotype of the CXCL9- and CXCL10-expressing CD45+ immune cells in the PbA-infected brain was analyzed by flow cytometry. The cortices of REX3 mice on day 8 p.i. were digested into single-cell suspensions and then passed through a Percoll gradient to enrich for leukocytes. There were easily identifiable CXCL10-BFP single-positive and CXCL10-BFP CXCL9-RFP double-positive populations in the brains of infected REX3 mice compared with the brains of infected WT mice. Figure 7A shows the gating strategy used for the data in $\mathrm{B}$ and $\mathrm{C}$ and the markers used to gate the populations for the data in D-G. Within the CXCL9-RFP/CXCL10-BFP double-positive cell population, $20 \%$ were microglia $\left(\mathrm{CD} 45^{\text {lo }} \mathrm{CD} 11 \mathrm{~b}^{+}\right)$and over $50 \%$ were mononuclear phagocytes $\left(\mathrm{CD} 45^{\mathrm{hi}} \mathrm{CD} 11 \mathrm{~b}^{+}\right)$. Upon further phenotyping, we found that $20 \%$ of the total CXCL9-RFP/CXCL10-BFP double-positive population were inflammatory monocytes (iMOs) $\left(\mathrm{CD} 45^{+} \mathrm{CD} 11 \mathrm{~b}^{+} \mathrm{CD} 11 \mathrm{c}^{-} \mathrm{Ly} 6 \mathrm{c}^{\mathrm{hi}}\right)$ and $20 \%$ were monocyte-

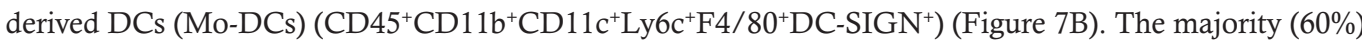
of the CXCL10-BFP single-positive population was microglia (Figure 7C). Less than 1\% of the Percollenriched single-cell suspension of cortex cells were CXCL9-RFP single-positive (Figure 7A), so these data were omitted from Figure 7, B and C.

We also gated on the cell subset first to determine what percentage of each subset produced CXCL9 and CXCL10 in the PbA-infected brain (Figure 7, D and E, white bars). In REX3 mice, the majority ( $\sim 65 \%)$ of mononuclear phagocytes were CXCL9/10 double-positive (Figure 7D, left), and of those, 80\% of iMOs and 70\% of Mo-DCs were CXCL9/10 double-positive (Figure 7D, right). Within the microglial population, few were CXCL9/10 double-positive ( 5\%) or CXCL10-BFP single-positive (10\%) (Figure 7, $\mathrm{D}$ and $\mathrm{E}$, white bars). This indicates that while a large percentage of the CXCL10-BFP single-positive cells were microglia, very few of the total population of microglia were CXCL10-BFP ${ }^{+}$, which is consistent with our whole-mount data (Figure 5). We also quantified the number of cells per cortex that were CXCL9/10 double-positive (Figure 7F, white bars) and CXCL10 single-positive (Figure 7G, white bars). Of the total population of CXCL9/10 double-positive cells, there were more mononuclear cells (iMOs plus Mo-DCs) than microglia (Figure 7F). Further, although there was a moderate number of CXCL10-BFP single-positive microglia (Figure 7G, left graph), this represented only $10 \%$ of the total microglia in the cortex (Figure $7 \mathrm{E}$, left graph). Of note, a comparison of CXCL10-BFP median fluorescence intensity in the populations of interest revealed that Mo-DCs (red line) had the highest level of expression, followed by iMOs (blue line), and microglia (black line) had a much lower level of CXCL10-BFP expression (Figure 7H).

We then quantified the percentage and number of CXCR3 ligand-positive cells in REX3-Ifnar ${ }^{\prime-}$ and REX3-Ifngr ${ }^{--}$mice (Figure 7, D-G). Consistent with the whole-mount and qPCR data in Figure 6, the percentage of CXCR3 ligand-positive cells were much lower in the REX-Ifngr/- mice, compared with REX3 or REX3-Ifnar ${ }^{-1}$ mice (Figure 7I). CXCL9/10 double-positive mononuclear phagocytes, and more specifically iMOs and Mo-DCs, were markedly decreased in REX3-Ifngr ${ }^{-1}$ (black bars) but not in REX3Ifnar ${ }^{\prime-}$ (gray bars) mice, compared with REX3 (white bars) mice (Figure 7D). In addition, although there was no difference in the percentage of these double-positive mononuclear phagocyte populations between REX3 and REX3-Ifnar ${ }^{-}$mice (Figure 7D), the overall number of double-positive mononuclear phagocytes per cortex was increased in the REX3-Ifnar ${ }^{--}$mice (Figure 7F), consistent with the qPCR data (Figure 6B). The percentage and number of CXCL10 single-positive microglia were also decreased in REX3-Ifngr ${ }^{\prime-1}$ mice (Figure 7, E and G). However, the percentage of CXCL10 single-producing iMOs and Mo-DCs were not affected in REX3-Ifngr ${ }^{\prime-}$ mice, but were slightly decreased in the cortex of REX3-Ifnar ${ }^{\prime-}$ mice (Figure $7 \mathrm{E})$. The number of these cells is so low, however, that they likely provide a relatively small fraction of the CXCL10-producing cells in the PbA-infected brain (Figure 7G).

EC CXCL10 increased T cell-EC adhesion strength and induced the onset of ECM. To specifically determine the role of EC-produced CXCL10 in T cell attachment and adhesion strength and the induction of ECM, we generated bone marrow chimeric mice. DPE-GFP bone marrow cells were injected into lethally irradiated $\mathrm{CxCl9^{-1- }}$ or $\mathrm{Cxcl10^{-- }}$ recipient mice and allowed to reconstitute for at least 8 weeks prior to infection with $\mathrm{PbA}$. DPE-GFP $\rightarrow$ WT and DPE-GFP $\mathrm{CxCr}^{-1-} \rightarrow \mathrm{Cxcr}^{-1-}$ chimeric mice were generated as radiation controls. One set of mice was observed for ECM onset and mortality following infection with $\mathrm{PbA}$ (Figure 8A). DPE$\mathrm{GFP} \rightarrow$ WT chimeric mice all succumbed to ECM by day 11 (Figure 8A, blue line), similar to nonirradiated $\mathrm{PbA}$-infected WT mice. The DPE-GFP $\mathrm{CxCr}^{-1-} \rightarrow \mathrm{CxCr}^{-1-}$ chimeric control mice were protected from ECM, with $55 \%$ mortality (Figure $8 \mathrm{~A}$, red line). This rate of protection was less than what we have previously observed for infected $\mathrm{CxCr}^{-/-}$mice (80\%), suggesting an effect of brain irradiation on survival. Nonetheless, 
we still found that there was an increase in the survival of DPE-GFP $\rightarrow C x c l 10^{-1-}$ mice (22\%), which have WT CXCL10-producing hematopoietic cells, but stromal cells that are deficient in CXCL10. Further, there was a delay in the onset of ECM mortality in the $\mathrm{Cxcl1}^{-/-}$chimera (Figure 8A, black line). The DPE-GFP $\rightarrow C x c 19^{-1-}$ chimeric mice did not have an increase in survival (Figure $8 \mathrm{~A}$, dashed line).

Using a separate set of chimeric mice, we performed MP-IVM to observe the effect of EC CXCR3 ligands on $\mathrm{T}$ cell interactions with the brain endothelium during ECM. The number of $\mathrm{T}$ cells in the vessel lumen tracked during IVM movie acquisition positively correlated with their mortality. WT and $\mathrm{Cxcl}^{-{ }^{-/}} \mathrm{chi}^{-}$ meras had the highest number of $\mathrm{T}$ cells interacting with the endothelium and the highest mortality, while $\mathrm{CxCr}^{-/-}$and $\mathrm{CxCl1}^{--}$chimeras had fewer T cells interacting with the endothelium and lower mortality (Figure $8 \mathrm{~B}$ ). In addition, $\mathrm{T}$ cells in the brains of mice that cannot produce CXCL10 in the stromal compartment had decreased arrest coefficients (Figure 8C) and increased proportion of detachment (Figure 8D). These data demonstrate that EC-produced CXCL10 in PbA-infected mice increases the strength of $\mathrm{T}$ cell attachment to the brain endothelium, leading to stronger T cell-EC adhesion (arrest coefficient), and a decrease in the percentage of attached $\mathrm{T}$ cells that subsequently detach. EC-derived CXCL10 is therefore a chemokine that plays an important role in the induction of ECM through modification of T cell-EC adhesion strength.

\section{Discussion}

We have used MP-IVM of live, anesthetized mice to define a role for CXCR3 and its ligand CXCL10 in $\mathrm{T}$ cell-mediated brain injury in ECM. Following $\mathrm{PbA}$ infection of $\mathrm{CxCr}^{-1-}$ mice, there were fewer $\mathrm{T}$ cells interacting with the vasculature (Figure $1 \mathrm{~B}$ and Supplemental Video 1), and the arrest coefficient was lower (Figure 1E) compared with T cells in infected WT mice. Further, the percentage of newly attached T cells that subsequently detached from the brain endothelium was higher in $\mathrm{PbA}$-infected $\mathrm{Cxcr} 3^{-1-}$ mice, compared with WT mice (Figure 2D and Supplemental Video 3). The majority of T cells interacting with the lumen of the brain vasculature were $\mathrm{CD}^{+}$(Figure $1 \mathrm{G}$ and Supplemental Video 2), which is consistent with previous studies showing that the majority of $\mathrm{T}$ cells in the brain and meninges in $\mathrm{PbA}$-infected mice are $\mathrm{CD}^{+} \mathrm{T}$ cells and depletion of these, but not $\mathrm{CD}^{+} \mathrm{T}$ cells, prevents $\mathrm{ECM}$ development (13). There was a small population of $\mathrm{CD}^{+}$cells that was also attached to the vessel wall. This is of interest, as $\mathrm{CD}^{+} \mathrm{T}$ cells have been described as initiators of subsequent $\mathrm{CD}^{+} \mathrm{T}$ cell migration to the brain (16). Using cotransfer of WT and $\mathrm{Cxcr}^{-1-} \mathrm{Ag}$-specific $\mathrm{CD}^{+} \mathrm{T}$ cells, we found that in the presence of full-blown ECM, there was still a deficiency in $\mathrm{Cxcr}^{--} \mathrm{Ag}$-specific $\mathrm{CD}^{+} \mathrm{T}$ cell interactions and accumulation in the brain (Figure $3, \mathrm{H}$ and I). The percentage of newly attached $C x c r 3^{-/-}$Ag-specific $\mathrm{CD}^{+} \mathrm{T}$ cells that subsequently detached was also increased compared with transferred WT Ag-specific CD8 $8^{+} \mathrm{T}$ cells (Figure $3 \mathrm{~L}$ ). This indicates the presence of a cell-intrinsic defect in the ability of the $\mathrm{CxCr}^{3--} \mathrm{T}$ cells to firmly attach to the brain endothelium. This finding was supported by short-term treatment of infected WT mice with CXCR3 ligand-blocking antibodies that decreased arrest coefficient (Figure 4A) and increased the detachment of newly adherent T cells (Figure 4D). These data provide what we believe to be the first in vivo demonstration that CXCR3 contributes to the strength of $\mathrm{T}$ cell-EC adhesion in a clinically relevant disease model and suggest that CXCL10 can be added to the small group of chemokines that have been demonstrated to function as arrest chemokines in vivo (50).

Using our REX3 reporter mouse (CXCL9 promoter driving RFP and CXCL10 promoter driving BFP), we found that CXCL10 was strongly and specifically upregulated in brain ECs in infected mice (Figure 5A and Supplemental Figure 3). We also found CXCL9 and CXCL10 promoter activity in CD45 cells within the vessel lumen of postcapillary and collecting venules in infected mouse brains (Figure 5B and Supplemental Figure 4). This pattern of CXCL9 and CXCL10 mRNA expression does completely agree with the different patterns of CXCL9 and CXCL10 protein expression detected by immunohistochemistry in this model in several prior publications $(6,7,37)$. However, the poor fidelity of these antibodies for immunohistochemistry led us to generate the CXCL9-CXCL10 dual reporter mice. This relatively new tool has been invaluable in robustly identifying cells that express CXCL9 and CXCL10 mRNA. The absence of CXCL10 expression on arterioles and its prominent expression on postcapillary and collecting venules in the brain is an interesting finding that likely reflects the specialization of the ECs that make up these different types of blood vessels. Intravascular leukocyte recruitment in most vertebrate tissues is restricted to postcapillary and collecting venules, whereas capillaries and arterioles usually support little or no leukocyte adhesion $(65,66)$. The prominent expression of chemokines and adhesion molecules in postcapillary and collecting venules that facilitate the capture, arrest, and transendothelial migration of leukocytes is a reflection of this 
specialized function. Using flow cytometry, we demonstrated that the majority of the CXCR3 ligand-producing immune cells were iMOs and Mo-DCs, whereas the majority of CXCL10 single-positive cells were microglia (Figure 7, B and C). However, only a small percentage of total microglia were producing CXCL10 (Figure 7E), while the majority of iMOs and Mo-DCs in the brain of infected mice were producing both CXCL9 and CXCL10 (Figure 7D). Although we also found a small percentage of microglia (10\%) that were CXCL10-BFP ${ }^{+}$by flow cytometry, their level of CXCL10-BFP expression was much lower than the other populations (Figure $7 \mathrm{H}$ ), perhaps explaining why they were not visualized by whole-mount microscopy or MP-IVM (Figure 5 and Supplemental Figure 5). Monocyte expression of CXCL10 is of interest, as it has been shown that monocytes accumulate along the brain endothelium, and depletion of these cells upon onset of ECM decreases the accumulation of $\mathrm{CD} 8^{+} \mathrm{T}$ cells in the brain $(67,68)$. Further, Mo-DCs have been recently shown to be one of the major CXCL9/10 producers in the spleen during the development of ECM. These cells were shown to traffic to the brain in a CCR5-dependent manner, possibly also contributing to the induction of $\mathrm{T}$ cell trafficking to the brain during the development of ECM by acting as early sources of CXCL9 and CXCL10 (69). Here, we have shown that there exists a population of iMOs and Mo-DCs firmly adhered to the lumen of the brain postcapillary venules, which produce high levels of both CXCL9 and -10 that are, in combination with EC-produced CXCL10, activating CXCR3 on the T cells in the blood and thus contributing to $\mathrm{T}$ cell-EC interactions within the brain during $\mathrm{CM}$.

ECM pathogenesis has been shown to be strongly dependent on $\operatorname{IFN}-\gamma(13,16,19,20)$. We found that IFN- $\gamma$ regulates the induction of CXCL9 and CXCL10 in brain ECs and immune cells in ECM (Figure 6A). Interestingly, we found that CXCL9 and CXCL10 were induced to higher levels in the brains of PbAinfected Ifnar ${ }^{-}$mice compared with WT mice (Figure 6B). Further, the number of CXCL9 and CXCL10 double-positive cells was higher in the brains of $\mathrm{PbA}$-infected Ifnar ${ }^{\prime-}$ mice compared with WT mice (Figure $7 \mathrm{~F})$. This is likely a result of an increase in IFN- $\gamma$ signaling caused by the absence of counterregulatory IFN- $\alpha / \beta$ receptor signaling, which has been described in some infectious settings $(70,71)$. The absence of CXCL10 production from the ECs and CXCL9 and CXCL10 from iMOs and Mo-DCs is likely one mechanism by which Ifng $^{-1-}$ mice are protected from $\mathrm{CM}$.

Using bone marrow chimeric mice, we demonstrated a functional role for EC-produced CXCL10 in the adhesion of T cells on the brain vasculature and the onset of lethal ECM (Figure 8, A and C). Although the increase in survival seen in mice lacking stromal CXCL10 was modest $(22 \%)$, we believe the disruption of the

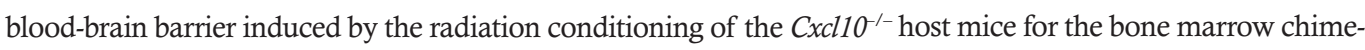
ras negatively influenced survival following infection. The effect of radiation on survival was also observed in the $\mathrm{CxCr}^{---} \rightarrow \mathrm{CxCr}^{---}$radiation control bone marrow chimeras, where the level of protection (55\%) was much lower than what was observed in unirradiated $\mathrm{Cxcr}^{-1-}$ mice (80\%) (6). The role of EC-produced CXCL10 is particularly interesting, as it has recently been shown that parasite-specific $\mathrm{CD} 8^{+} \mathrm{T}$ cells interact in a peptideMHC I-dependent manner with the brain vascular EC lumen, where they were observed surveying, dividing, and arresting (13). As with CXCR3 ligand production, these activities were shown to be IFN- $\gamma$ dependent, leading to EC activation, including adhesion and antigen-presentation-molecule upregulation. Here we have shown a role for IFN- $\gamma$-induced EC-produced CXCL10 in inducing long-lasting CD8 ${ }^{+} \mathrm{T}$ cell adhesion on the luminal surface of brain vasculature in PbA-infected mice (Supplemental Figure 6). Our data therefore suggest that the CXCL10/CXCR3 pathway facilitates the interaction of CD8 ${ }^{+} \mathrm{T}$ cells with cognate peptide-MHC I complexes on the luminal surface of the activated brain endothelium. Our data also provide a mechanistic framework and further rationale for inhibiting the CXCR3 chemokine system to prevent or possibly treat CM. This is particularly pertinent with the emergence of insecticide-resistant mosquitos and artemisinin-resistant parasites $(1,2)$.

\section{Methods}

Mice. C57BL/6 WT mice were purchased from the National Cancer Institute, National Institutes of Health $(\mathrm{NIH})$, with the exception of the OT-I transfer recipients, which were CD45.1 congenic C57BL/6 mice purchased from The Jackson Laboratory. DPE-GFP mice (56) were a gift from Ulrich H. von Andrian (Harvard Medical School, Boston, Massachusetts, USA). CXCR3-deficient ( $\mathrm{Cxcr}^{-/-}$) mice (72) were a gift from Craig Gerard (Children's Hospital, Boston, Massachusetts, USA), Cxcl9 ${ }^{-/-}$mice (73) were a gift from Joshua M. Farber (NIAID, NIH, Bethesda, Maryland, USA), and the Ifnar ${ }^{-1}$ mice (74) were a gift from Michel Aguet (University of Zurich, Zurich, Switzerland). $C x \mathrm{Cl1}^{-/-}$mice were generated in our laboratory and backcrossed onto C57BL/6 for 11 generations (75). $C x c r 3^{-/-}$mice were received backcrossed 10 generations, and have been backcrossed to 12 total in our laboratory. $\mathrm{Cxcl9^{-1- }}$ mice were received backcrossed 
8 generations, and have been backcrossed to a total of 10 generations in our laboratory. REX3 mice were generated by our lab in C57BL/6 mice (58). Ifngr ${ }^{-1-}$, Actin-CFP, Actin-TdTomato, and OT-I breeders were purchased from The Jackson Laboratory. $\mathrm{CxCr}^{-/-}, \mathrm{CxCl9^{-- }}, \mathrm{CxCl10^{-- }}$, and REX3 mouse strains were rederived to be free of Helicobacter and Pasteurella pneumotropica.

Infections and survival curves. Eight- to 12 -week-old male and female mice were injected i.p. with $1 \times 10^{6}$ to $2 \times 10^{6} \mathrm{PbA}$-infected $\mathrm{pRBC}$. Mice in OT-I transfer experiments were infected 2 hours after $\mathrm{T}$ cell transfer with $\mathrm{PbA}-\mathrm{OVA}$ pRBC i.p. (76). For survival curves, mortality was checked twice daily, and mice showing signs of severe CM (ataxia, convulsion, coma) between days 6 and 10 after infection were sacrificed. Mice without CM developed anemia because of rising parasitemia and were sacrificed on day 20. Endpoints other than mortality are described below.

Cranial window surgery and brain MP-IVM. Unless otherwise stated, 8 or 9 days p.i., mice were anesthetized with ketamine/xylazine injected i.p. and cranial window surgery was performed as described in the Supplemental Methods (77). The brain vasculature was visualized by r.o. injection of nontargeted quantum dots, QTracker 655 (Thermo Fisher Scientific). Movies were acquired using multiphoton microscopes further described in Supplemental Methods. T cell migration was analyzed and movies produced using Imaris (Bitplane). Arrest coefficients were calculated using custom scripts written for MATLAB (MathWorks) software (78) and defined as the proportion of time during which an individual tracked $\mathrm{T}$ cell moves with an instantaneous velocity below $2 \mu \mathrm{m} / \mathrm{min}$. Attachment and detachment were counted by eye; attachment was considered any newly visualized $\mathrm{T}$ cell that stayed immobile on the vessel lumen for 2 frames (longer than 30 seconds). The marked increase in T cell-EC interactions seen in infected DPE-GFP mice was a response to $\mathrm{PbA}$ infection and not the cranial window surgery, as the number of T cells per FOV was markedly lower in uninfected DPE-GFP mice (Figure 1B).

In order to stain for $\mathrm{CD}^{+}$and $\mathrm{CD}^{+}$cells, briefly, cranial windows were installed in WT mice, and anti-CD4 and anti-CD8 mAbs conjugated to the indicated fluorochromes were injected r.o. 2 hours prior to imaging by MP-IVM, as described in the Supplemental Methods. For the antibody treatment MP-IVM experiments, infected mice were imaged on days 8 or 9 , as described above, and then $200 \mu \mathrm{g}$ anti-CXCL9 (79), anti-CXCL10 (clone 1F11) (80), or hamster IgG control (BioLegend) was injected r.o., and after 4 hours the same FOV was imaged again.

$T$ cell transfer. Spleens and peripheral lymph nodes of naive Actin-TdTomato $\mathrm{Cxcr}^{+/+}$OT-I mice or Actin-CFP $\mathrm{Cxcr}^{-/-}$OT-I mice were harvested and T cells isolated as described in the Supplemental Methods. For flow cytometry experiments, $10^{5}$ of each genotype, or $5 \times 10^{5}$ of each genotype for MP-IVM experiments, were then mixed at a 1:1 ratio and injected r.o. into CD45.1 $1^{+}$WT recipient mice. Mice were infected with PbA-OVA pRBC 2 hours after cell transfer, as described above.

Flow cytometric analysis. Detailed methodology is described in the Supplemental Methods. Briefly, for $\mathrm{T}$ cell transfer experiments, on day 8 p.i., spleen, blood, and brain were isolated and processed for FACS staining. Spleens were processed using a 70- $\mu \mathrm{m}$ cell strainer and RBC lysed using RBC lysing agent (Sigma-Aldrich) and brain cells dissociated into a single-cell suspension and non-myelinated cells isolated using a Percoll gradient. Cells were then stained with the indicated antibodies or appropriate isotype control.

For REX3 brain flow cytometry, brains were removed after intracardial perfusion and cerebral cortices were isolated and processed into a single-cell suspension as described in the Supplemental Methods. Cells were blocked and stained with the indicated antibodies or appropriate isotype control.

Whole-mount histology. Whole mount of brain tissue was performed as previously described (81). Briefly, cerebral cortices were dissected as described in the Supplemental Methods and small pieces of brain cortex were blocked and stained with the indicated fluorochrome-conjugated mAbs. Filters designed to detect AF488, RFP, APC, and BFP wavelengths were used to acquire alternate images of the same FOVs. Monochrome images were acquired and pseudocolored using Zen Blue software (Zeiss).

Quantitative PCR. Brain cortices, dissected as described above, and spleens of mice, either uninfected or at different time points after infection with $\mathrm{PbA}$, were removed after intracardial perfusion with $20 \mathrm{ml}$ of PBS, 1 mM EDTA. Total RNA was isolated by using TRIzol and subsequently with an RNAeasy kit (Qiagen) for brain homogenate or TRIzol only for spleen homogenate. Total RNA was converted to cDNA and analyzed for CXCL9 and CXCL10 by qPCR as previously described (82) using the LightCycler 96 (Roche) quantitative PCR system. 
Generation of bone marrow chimeric mice. Bone marrow was isolated from femurs of DPE-GFP or DPE-GFP $\mathrm{CXCr}^{---}$mice using a mortar and pestle, passed through a $70-\mu \mathrm{m}$ strainer, and washed in PBS. Bone marrow cells $\left(3 \times 10^{6}\right)$ in PBS were injected i.v. via tail vein into lethally total-body irradiated (10 Gy) WT, $C x C l 9^{--}, C x c l 10^{--}$, or $C x \mathrm{Cr}^{-/-}$recipient mice on the same day. After injection, chimeric mice were allowed to reconstitute for a minimum of 8 weeks before experimental use. Percentage chimerism was measured in all chimeras by the percentage of $\mathrm{GFP}^{+}$circulating $\mathrm{T}$ cells, and only chimeras that had $80 \%$ or higher donor $\mathrm{T}$ cell populations were used.

Statistics. The sample size was determined to achieve a power of $90 \%$, accepting a type I error rate of 0.05. Randomization and blinding were not used for analysis of movies; however, a standardized autotracking protocol was applied to all movies and then the tracking completed manually. Statistical analysis was performed by unpaired, nonparametric 2-tailed Mann-Whitney $t$ test or a 1-way Kruskal-Wallis ANOVA with Dunn's multiple comparison test, in the case of IVM experiments, with the exception of the data in Figure 1G; Figure 3, B, J-L; and Figure 4, in which a paired nonparametric Wilcoxon test was used. A paired, nonparametric 1-way Friedman's ANOVA with Dunn's multiple comparison test was used in Figure 3, C-I. Unpaired 2-way ANOVA with Bonferroni's or Sidak's multiple comparison test for Figure 6, B and $\mathrm{C}$ and 7, depending on if comparing between mouse strain or time point, and by Mantel-Cox log-rank test for differences in mortality. Violin plots contain box plots that display the median, 25 th and 75 th percentiles, and whiskers that are equal to $(1.58 \times$ interquartile range $) / \operatorname{sqrt}(n)$ or the $95 \%$ confidence interval. For all tests, a $P$ value of less than 0.05 was considered significant.

Study approval. All crosses mentioned in the Results section were bred at Massachusetts General Hospital. All protocols were approved by the Massachusetts General Hospital Subcommittee on Research and Animal Care and adhere to the NIH Guide for the Care and Use of Laboratory Animals.

\section{Author contributions}

EWS designed and performed experiments in all figures, with the exception of Figure 3; Figure 6, B and C; Figure 7; and Supplemental Figure 2, C and D; evaluated and interpreted data, and wrote the manuscript. JL performed qPCR in Figure 6, B and C and Supplemental Figure 2, C and D and brain flow cytometry in Figure 7 and evaluated the data. AJO performed the experiments in Figure 2 and evaluated the data. YM evaluated and interpreted attachment and detachment data in all MP-IVM experiments. SKB produced all bone marrow chimeric mice with EWS. SWJ performed qPCR with JL and tracked most of the videos from MP-IVM experiments run by EWS. TRM provided technical support and guidance with the 2-photon microscopes. ADL designed and supervised the study and contributed to writing the manuscript. All authors edited the manuscript.

\section{Acknowledgments}

This work was supported by NIH grants R01NS098747, R01CA204028, T32HL007874, and T32AR007258 to A.D. Luster. We would also like to thank Esteban Carrizosa for assistance with MP-IVM and Francesco Marangoni for assistance with statistical methodologies.

Address correspondence to: Andrew D. Luster, Center for Immunology and Inflammatory Diseases, Massachusetts General Hospital, CNY 149-8, 149 13th Street, Charlestown, Massachusetts 02129, USA. Phone: 617.724.6147; Email: aluster@mgh.harvard.edu.

1. World Malaria Report 2016. World Health Organization (WHO). http://www.who.int/malaria/publications/world-malariareport-2016/report/en/. Accessed March 28, 2018

2. Phillips MA, Burrows JN, Manyando C, van Huijsduijnen RH, Van Voorhis WC, Wells TNC. Malaria. Nat Rev Dis Primers. 2017;3:17050

3. Murphy SC, Breman JG. Gaps in the childhood malaria burden in Africa: cerebral malaria, neurological sequelae, anemia, respiratory distress, hypoglycemia, and complications of pregnancy. Am J Trop Med Hyg. 2001;64(1-2 Suppl):57-67.

4. Bauer PR, Van Der Heyde HC, Sun G, Specian RD, Granger DN. Regulation of endothelial cell adhesion molecule expression in an experimental model of cerebral malaria. Microcirculation. 2002;9(6):463-470.

5. Chang WL, et al. P-selectin contributes to severe experimental malaria but is not required for leukocyte adhesion to brain microvasculature. Infect Immun. 2003;71(4):1911-1918.

6. Campanella GS, et al. Chemokine receptor CXCR3 and its ligands CXCL9 and CXCL10 are required for the development of murine cerebral malaria. Proc Natl Acad Sci USA. 2008;105(12):4814-4819.

7. Miu J, et al. Chemokine gene expression during fatal murine cerebral malaria and protection due to CXCR3 deficiency. $J$ Immu- 
nol. 2008;180(2):1217-1230.

8. Belnoue E, et al. CCR5 deficiency decreases susceptibility to experimental cerebral malaria. Blood. 2003;101(11):4253-4259.

9. Dunst J, Kamena F, Matuschewski K. Cytokines and chemokines in cerebral malaria pathogenesis. Front Cell Infect Microbiol. 2017;7:324.

10. Nacer A, Movila A, Baer K, Mikolajczak SA, Kappe SH, Frevert U. Neuroimmunological blood brain barrier opening in experimental cerebral malaria. PLoS Pathog. 2012;8(10):e1002982.

11. Nacer A, et al. Experimental cerebral malaria pathogenesis--hemodynamics at the blood brain barrier. PLoS Pathog. 2014;10(12):e1004528.

12. Mota MM, Jarra W, Hirst E, Patnaik PK, Holder AA. Plasmodium chabaudi-infected erythrocytes adhere to CD36 and bind to microvascular endothelial cells in an organ-specific way. Infect Immun. 2000;68(7):4135-4144.

13. Swanson $\mathrm{PA}$, et al. $\mathrm{CD}^{+} \mathrm{T}$ cells induce fatal brainstem pathology during cerebral malaria via luminal antigen-specific engagement of brain vasculature. PLoS Pathog. 2016;12(12):e1006022.

14. Belnoue $\mathrm{E}$, et al. On the pathogenic role of brain-sequestered alphabeta $\mathrm{CD} 8^{+} \mathrm{T}$ cells in experimental cerebral malaria. $J$ Immunol. 2002;169(11):6369-6375.

15. Hermsen C, van de Wiel T, Mommers E, Sauerwein R, Eling W. Depletion of CD4 ${ }^{+}$or CD8 $8^{+}$T-cells prevents Plasmodium berghei induced cerebral malaria in end-stage disease. Parasitology. 1997;114(Pt 1):7-12.

16. Villegas-Mendez A, et al. IFN- $\gamma$-producing CD4 ${ }^{+} \mathrm{T}$ cells promote experimental cerebral malaria by modulating $\mathrm{CD} 8^{+} \mathrm{T}$ cell accumulation within the brain. J Immunol. 2012;189(2):968-979.

17. Nitcheu J, et al. Perforin-dependent brain-infiltrating cytotoxic CD ${ }^{+} \mathrm{T}$ lymphocytes mediate experimental cerebral malaria pathogenesis. J Immunol. 2003;170(4):2221-2228.

18. Haque A, et al. Granzyme B expression by $\mathrm{CD} 8^{+} \mathrm{T}$ cells is required for the development of experimental cerebral malaria. J Immunol. 2011;186(11):6148-6156

19. Amani V, et al. Involvement of IFN-gamma receptor-medicated signaling in pathology and anti-malarial immunity induced by Plasmodium berghei infection. Eur J Immunol. 2000;30(6):1646-1655.

20. Belnoue E, et al. Control of pathogenic $\mathrm{CD}^{+} \mathrm{T}$ cell migration to the brain by IFN-gamma during experimental cerebral malaria. Parasite Immunol. 2008;30(10):544-553.

21. Howland SW, et al. Brain microvessel cross-presentation is a hallmark of experimental cerebral malaria. EMBO Mol Med. 2013;5(7):984-999.

22. Howland SW, Poh CM, Rénia L. Activated brain endothelial cells cross-present malaria antigen. PLoS Pathog. 2015;11(6):e1004963.

23. Lucchi NW, Jain V, Wilson NO, Singh N, Udhayakumar V, Stiles JK. Potential serological biomarkers of cerebral malaria. Dis Markers. 2011;31(6):327-335.

24. Jain V, et al. Plasma IP-10, apoptotic and angiogenic factors associated with fatal cerebral malaria in India. Malar J. 2008;7:83.

25. Armah HB, et al. Cerebrospinal fluid and serum biomarkers of cerebral malaria mortality in Ghanaian children. Malar J. 2007;6:147

26. Wilson NO, et al. CXCL4 and CXCL10 predict risk of fatal cerebral malaria. Dis Markers. 2011;30(1):39-49.

27. Wilson N, et al. CXCL10 gene promoter polymorphism -1447A $>$ G correlates with plasma CXCL10 levels and is associated with male susceptibility to cerebral malaria. PLoS ONE. 2013;8(12):e81329.

28. Luster AD, Unkeless JC, Ravetch JV. Gamma-interferon transcriptionally regulates an early-response gene containing homology to platelet proteins. Nature. 1985;315(6021):672-676.

29. Loetscher M, et al. Chemokine receptor specific for IP10 and mig: structure, function, and expression in activated T-lymphocytes. J Exp Med. 1996;184(3):963-969.

30. Farber JM. A macrophage mRNA selectively induced by gamma-interferon encodes a member of the platelet factor 4 family of cytokines. Proc Natl Acad Sci USA. 1990;87(14):5238-5242.

31. Cole KE, et al. Interferon-inducible T cell alpha chemoattractant (I-TAC): a novel non-ELR CXC chemokine with potent activity on activated T cells through selective high affinity binding to CXCR3. J Exp Med. 1998;187(12):2009-2021.

32. Sierro F, et al. Disrupted cardiac development but normal hematopoiesis in mice deficient in the second CXCL12/SDF-1 receptor, CXCR7. Proc Natl Acad Sci USA. 2007;104(37):14759-14764.

33. Rabin RL, et al. CXCR3 is induced early on the pathway of $\mathrm{CD}^{+} \mathrm{T}$ cell differentiation and bridges central and peripheral functions. J Immunol. 2003;171(6):2812-2824.

34. Qin S, et al. The chemokine receptors CXCR3 and CCR5 mark subsets of T cells associated with certain inflammatory reactions. J Clin Invest. 1998;101(4):746-754.

35. Lord GM, et al. T-bet is required for optimal proinflammatory CD4+ T-cell trafficking. Blood. 2005;106(10):3432-3439.

36. Taqueti VR, et al. T-bet controls pathogenicity of CTLs in the heart by separable effects on migration and effector activity. J Immunol. 2006;177(9):5890-5901.

37. Van den Steen PE, et al. CXCR3 determines strain susceptibility to murine cerebral malaria by mediating T lymphocyte migration toward IFN-gamma-induced chemokines. Eur J Immunol. 2008;38(4):1082-1095.

38. Campanella GS, Medoff BD, Manice LA, Colvin RA, Luster AD. Development of a novel chemokine-mediated in vivo T cell recruitment assay. J Immunol Methods. 2008;331(1-2):127-139.

39. Campanella GS, et al. Oligomerization of CXCL10 is necessary for endothelial cell presentation and in vivo activity. $J$ Immunol. 2006;177(10):6991-6998.

40. Groom JR, Luster AD. CXCR3 in T cell function. Exp Cell Res. 2011;317(5):620-631.

41. Groom JR, Luster AD. CXCR3 ligands: redundant, collaborative and antagonistic functions. Immunol Cell Biol. 2011;89(2):207-215.

42. Nie CQ, et al. IP-10-mediated T cell homing promotes cerebral inflammation over splenic immunity to malaria infection. PLoS Pathog. 2009;5(4):e1000369.

43. Jeyanathan $\mathrm{M}$, et al. CXCR3 signaling is required for restricted homing of parenteral tuberculosis vaccine-induced $\mathrm{T}$ cells to both the lung parenchyma and airway. J Immunol. 2017;199(7):2555-2569.

44. Kohlmeier JE, et al. Inflammatory chemokine receptors regulate CD8(+) T cell contraction and memory generation following 
infection. JExp Med. 2011;208(8):1621-1634.

45. Kurachi M, et al. Chemokine receptor CXCR3 facilitates CD8(+) T cell differentiation into short-lived effector cells leading to memory degeneration. J Exp Med. 2011;208(8):1605-1620.

46. Hu JK, Kagari T, Clingan JM, Matloubian M. Expression of chemokine receptor CXCR3 on T cells affects the balance between effector and memory CD8 T-cell generation. Proc Natl Acad Sci USA. 2011;108(21):E118-E127.

47. Drennan MB, et al. Cutting edge: the chemokine receptor CXCR3 retains invariant NK T cells in the thymus. $J$ Immunol. 2009;183(4):2213-2216.

48. Mackay LK, et al. The developmental pathway for CD103(+)CD8+ tissue-resident memory T cells of skin. Nat Immunol. 2013;14(12):1294-1301.

49. Sathiyanadan K, Coisne C, Enzmann G, Deutsch U, Engelhardt B. PSGL-1 and E/P-selectins are essential for T-cell rolling in inflamed CNS microvessels but dispensable for initiation of EAE. Eur J Immunol. 2014;44(8):2287-2294.

50. Ley K. Arrest chemokines. Front Immunol. 2014;5:150.

51. Stein JV, et al. The CC chemokine thymus-derived chemotactic agent 4 (TCA-4, secondary lymphoid tissue chemokine, 6 Ckine, exodus-2) triggers lymphocyte function-associated antigen 1-mediated arrest of rolling $\mathrm{T}$ lymphocytes in peripheral lymph node high endothelial venules. JExp Med. 2000;191(1):61-76.

52. Piali L, et al. The chemokine receptor CXCR3 mediates rapid and shear-resistant adhesion-induction of effector T lymphocytes by the chemokines IP10 and Mig. Eur J Immunol. 1998;28(3):961-972.

53. D'Ambrosio D, Albanesi C, Lang R, Girolomoni G, Sinigaglia F, Laudanna C. Quantitative differences in chemokine receptor engagement generate diversity in integrin-dependent lymphocyte adhesion. J Immunol. 2002;169(5):2303-2312.

54. Bartholomäus I, et al. Effector T cell interactions with meningeal vascular structures in nascent autoimmune CNS lesions. Nature. 2009;462(7269):94-98.

55. Shaw TN, et al. Perivascular arrest of $\mathrm{CD}^{+} \mathrm{T}$ cells is a signature of experimental cerebral malaria. PLoS Pathog 2015;11(11):e1005210.

56. Mempel TR, et al. Regulatory T cells reversibly suppress cytotoxic T cell function independent of effector differentiation. Immunity. 2006;25(1):129-141.

57. Abtin A, et al. Perivascular macrophages mediate neutrophil recruitment during bacterial skin infection. Nat Immunol. 2014;15(1):45-53.

58. Groom JR, et al. CXCR3 chemokine receptor-ligand interactions in the lymph node optimize CD4 $4^{+} \mathrm{T}$ helper 1 cell differentiation. Immunity. 2012;37(6):1091-1103.

59. Kretschmer S, et al. Autofluorescence multiphoton microscopy for visualization of tissue morphology and cellular dynamics in murine and human airways. Lab Invest. 2016;96(8):918-931.

60. Bechmann I, Galea I, Perry VH. What is the blood-brain barrier (not)? Trends Immunol. 2007;28(1):5-11.

61. Murooka TT, et al. HIV-infected T cells are migratory vehicles for viral dissemination. Nature. 2012;490(7419):283-287.

62. Ball EA, et al. IFNAR1 controls progression to cerebral malaria in children and $\mathrm{CD}^{+} \mathrm{T}$ cell brain pathology in Plasmodium berghei-infected mice. J Immunol. 2013;190(10):5118-5127.

63. Palomo J, et al. Type I interferons contribute to experimental cerebral malaria development in response to sporozoite or bloodstage Plasmodium berghei ANKA. Eur J Immunol. 2013;43(10):2683-2695.

64. Sharma S, et al. Innate immune recognition of an AT-rich stem-loop DNA motif in the Plasmodium falciparum genome. Immunity. 2011;35(2):194-207.

65. Thiriot A, et al. Differential DARC/ACKR1 expression distinguishes venular from non-venular endothelial cells in murine tissues. BMC Biol. 2017;15(1):45

66. von Andrian UH. Intravital microscopy of the peripheral lymph node microcirculation in mice. Microcirculation. 1996;3(3):287-300.

67. Ioannidis LJ, Nie CQ, Ly A, Ryg-Cornejo V, Chiu CY, Hansen DS. Monocyte- and neutrophil-derived CXCL10 impairs efficient control of blood-stage malaria infection and promotes severe disease. J Immunol. 2016;196(3):1227-1238.

68. Pai S, et al. Real-time imaging reveals the dynamics of leukocyte behaviour during experimental cerebral malaria pathogenesis PLoS Pathog. 2014;10(7):e1004236.

69. Hirako IC, et al. Splenic differentiation and emergence of CCR5 ${ }^{+}$CXCL9 ${ }^{+}$CXCL $10^{+}$monocyte-derived dendritic cells in the brain during cerebral malaria. Nat Commun. 2016;7:13277.

70. Nguyen KB, Cousens LP, Doughty LA, Pien GC, Durbin JE, Biron CA. Interferon alpha/beta-mediated inhibition and promotion of interferon gamma: STAT1 resolves a paradox. Nat Immunol. 2000;1(1):70-76.

71. Nguyen KB, et al. Critical role for STAT4 activation by type 1 interferons in the interferon-gamma response to viral infection. Science. 2002;297(5589):2063-2066.

72. Hancock WW, et al. Requirement of the chemokine receptor CXCR3 for acute allograft rejection. J Exp Med. 2000;192(10):1515-1520.

73. Park MK, et al. The CXC chemokine murine monokine induced by IFN-gamma (CXC chemokine ligand 9) is made by APCs, targets lymphocytes including activated B cells, and supports antibody responses to a bacterial pathogen in vivo. J Immunol. 2002;169(3):1433-1443.

74. Müller U, et al. Functional role of type I and type II interferons in antiviral defense. Science. 1994;264(5167):1918-1921.

75. Dufour JH, Dziejman M, Liu MT, Leung JH, Lane TE, Luster AD. IFN-gamma-inducible protein 10 (IP-10; CXCL10)-deficient mice reveal a role for IP-10 in effector T cell generation and trafficking. J Immunol. 2002;168(7):3195-3204.

76. Lundie RJ, et al. Blood-stage Plasmodium infection induces $\mathrm{CD} 8^{+} \mathrm{T}$ lymphocytes to parasite-expressed antigens, largely regulated by CD8alpha+ dendritic cells. Proc Natl Acad Sci USA. 2008;105(38):14509-14514.

77. Meyer-Luehmann M, et al. Rapid appearance and local toxicity of amyloid-beta plaques in a mouse model of Alzheimer's disease. Nature. 2008;451(7179):720-724.

78. Marangoni F, et al. The transcription factor NFAT exhibits signal memory during serial $\mathrm{T}$ cell interactions with antigen-presenting cells. Immunity. 2013;38(2):237-249.

79. Zeng $\mathrm{X}$, et al. Interferon-inducible protein 10 , but not monokine induced by gamma interferon, promotes protective type 1 immunity in murine Klebsiella pneumoniae pneumonia. Infect Immun. 2005;73(12):8226-8236. 
80. Khan IA, et al. IP-10 is critical for effector T cell trafficking and host survival in Toxoplasma gondii infection. Immunity. 2000;12(5):483-494

81. Gerber SA, Moran JP, Frelinger JG, Frelinger JA, Fenton BM, Lord EM. Mechanism of IL-12 mediated alterations in tumour blood vessel morphology: analysis using whole-tissue mounts. Br J Cancer. 2003;88(9):1453-1461.

82. Means TK, Hayashi F, Smith KD, Aderem A, Luster AD. The Toll-like receptor 5 stimulus bacterial flagellin induces maturation and chemokine production in human dendritic cells. J Immunol. 2003;170(10):5165-5175. 